\title{
Topological Crystalline Materials of $J=3 / 2$ Electrons: Antiperovskites, Dirac Points, and High Winding Topological Superconductivity
}

\author{
Takuto Kawakami, ${ }^{1, *}$ Tetsuya Okamura, ${ }^{1}$ Shingo Kobayashi, ${ }^{2,3}$ and Masatoshi Sato ${ }^{1, \dagger}$ \\ ${ }^{1}$ Yukawa Institute for Theoretical Physics, Kyoto University, Kyoto 606-8502, Japan \\ ${ }^{2}$ Institute for Advanced Research, Nagoya University, Nagoya 464-8601, Japan \\ ${ }^{3}$ Department of Applied Physics, Nagoya University, Nagoya 464-8603, Japan
}

(Received 4 March 2018; revised manuscript received 6 August 2018; published 13 November 2018)

\begin{abstract}
We present a theory of the high-spin generalization of topological insulators and their doped superconducting states. The higher-spin topological insulators involve a pair of $J=3 / 2$ bands with opposite parity and are characterized by their band inversion. The low-energy effective theory reveals that the topological insulators host four different phases characterized by mirror Chern numbers, at which boundaries two different patterns of bulk Dirac points appear. For the carrier-doped case, it is shown that the system may host unique unconventional superconductivity because of its high-spin nature and additional orbital degrees of freedom intrinsic to topological insulators. The superconducting critical temperature is evaluated by using density-density pairing interactions, and odd-parity Cooper pairs are shown to be naturally realized in the presence of interorbital pairing interaction. It is observed that even the simplest spin-0 odd-parity pairing state exhibits a novel class of topological superconductivity-high winding topological superconductivity. We also discuss the experimental signals of high winding topological superconductivity in the case of the antiperovskite superconductor $\mathrm{Sr}_{3-x} \mathrm{SnO}$.

DOI: 10.1103/PhysRevX.8.041026

\author{
Subject Areas: Condensed Matter Physics, \\ Superconductivity, \\ Topological Insulators
}

\section{INTRODUCTION}

The search for topological materials is a recent trend in condensed matter physics [1-8]. A promising direction to fulfill this search is a multiorbital material with strong spinorbit coupling. Such a system can be topologically nontrivial through band inversion owing to spin-orbit coupling. For instance, in the case of topological insulators (TIs), the $\boldsymbol{Z}_{2}$ topological indices are directly related to the number of timereversal-invariant momenta at which band inversion occurs $[9,10]$. Following this insight, numerous TIs including $\mathrm{Bi}_{2} \mathrm{Se}_{3}$ and $\mathrm{Bi}_{2} \mathrm{Te}_{3}$ have been discovered experimentally $[1,4]$.

In the case of superconductors, even a single orbital system may possess a nontrivial topological phase, because the charge-conjugate counterpart of the single band coexists in the Bogoliubov-de Gennes (BdG) Hamiltonian [11-17]. Any spin-triplet superconductor can be topological if the Fermi surface is properly chosen [17]. However,

\footnotetext{
takuto.kawakami@yukawa.kyoto-u.ac.jp

msato@yukawa.kyoto-u.ac.jp
}

Published by the American Physical Society under the terms of the Creative Commons Attribution 4.0 International license. Further distribution of this work must maintain attribution to the author(s) and the published article's title, journal citation, and DOI. the search for spin-triplet superconductivity itself is a challenge because of its strongly correlated origin. No spin-triplet superconductor has been established yet, despite the existence of several promising candidates.

Recently, it has been recognized that multiorbital systems may solve this difficulty, as they allow another mechanism of topological superconductivity [8]: Using interorbital pairing interaction and spin-orbit coupling, a multiorbital system may host odd-parity Cooper pairs even without a strong correlation [18], which indicates topological superconductivity [17-19]. In this new direction, various types of topological superconductivity in doped topological materials have been discussed recently [18,20-37].

In this study, we explore another class of topological phases that can be realized only in $J=3 / 2$ bands. Spinorbit coupled electrons may behave as higher-order spin states, owing to the mixture of spin and orbital angular momentum. In crystals, the higher-spin state can be identified as a $J=3 / 2$ state, as discrete crystalline rotation allows at most fourfold degeneracy of the $J=3 / 2$ spin. Superconductivity of $J=3 / 2$ electrons has been discussed for Luttinger semimetals with a single $J=3 / 2$ band such as half-Heusler systems [38-49]. While the half-Heusler systems may host interesting higher-spin Cooper pairs, their actual realization is restricted, because the systems support only a single $J=3 / 2$ band. 
Here, alternatively, we consider systems with multiple $J=3 / 2$ bands. A class of relevant materials is antiperovskite $A_{3} B X$, where $A$ is $(\mathrm{Ca}, \mathrm{Sr}, \mathrm{La}), B$ is $(\mathrm{Pb}, \mathrm{Sn})$, and $X$ is $(\mathrm{C}, \mathrm{N}, \mathrm{O})$. The antiperovskite materials support two different $J=3 / 2$ electrons near the Fermi level, i.e., $d$-orbital electrons of the $A$ atom and $p$-orbital ones of the $B$. The first-principles calculations show that the band inversion of these two orbitals may occur at the $\Gamma$ point, accompanied by three-dimensional (3D) Dirac points with a tiny gap [50-52]. Owing to the band inversion, these materials become topological crystalline insulators [52,53]. It was also discovered very recently that one of the antiperovskite topological materials, $\mathrm{Sr}_{3} \mathrm{SnO}$, shows superconductivity with hole doping [54-58]. Although a discussion from the viewpoint of $J=3 / 2$ electrons is missing, possible topological superconductivity in $\mathrm{Sr}_{3-x} \mathrm{SnO}$ is suggested theoretically [54], using the analogy of the superconducting Dirac semimetal $\mathrm{Cd}_{3} \mathrm{As}_{2}[35,36]$.

Herein, we develop a general theory of higher-spin topological materials. Considering the application to antiperovskites, we assume cubic symmetry, which allows for four-dimensional representation corresponding to the fourfold degeneracy of $J=3 / 2$. Based on the low-energy effective Hamiltonian, we observe that the system hosts four different topological crystalline insulating phases, where the phase boundaries are characterized by two different patterns of gapless Dirac points. In addition to the octahedral Dirac points discussed previously [50-52], cubic Dirac points appear at one of the phase boundaries.

We thereafter study the superconducting states of doped higher-spin topological materials. We first classify possible momentum-independent gap functions, based on the cubic symmetry. The gap functions may contain spin $J=2$ (spin-quintet) and $J=3$ (spin-septet) components and ordinary $J=0$ (spin-singlet) and $J=1$ (spin-triplet) ones, as Cooper pairs are formed by the $J=3 / 2$ electrons. In contrast to the single $J=3 / 2$ band case as in half-Heusler materials, additional orbital degrees of freedom (d.o.f.) make it possible to obtain any higher-spin gap function in the framework of weakly correlated constant gap functions. Subsequently, we evaluate the superconducting critical temperature $T_{c}$ for each gap function by using simple density-density pairing interactions. It is observed that the system supports odd-parity superconductivity if the interorbital pairing interaction is dominant. The $J=0$ oddparity pairing state with $A_{1 u}$ representation yields the highest $T_{c}$ among odd-parity superconducting states, but the $T_{1 u}$ pairing state consisting of $J=3$ and $J=1$ Cooper pairs also has high $T_{c}$.

We reveal that the simplest $J=0$ odd-parity pairing state shows a new class of topological superconductivity, i.e., high winding topological superconductivity. As a 3D timereversal-invariant superconductor, the odd-parity pairing state has a nonzero $3 \mathrm{D}$ winding number similar to the ${ }^{3} \mathrm{He}-\mathrm{B}$ phase. However, in contrast to ${ }^{3} \mathrm{He}-\mathrm{B}$, owing to the higher-spin nature of $J=3 / 2$ electrons, it does not support the minimal nonzero value but hosts various higher winding numbers depending on the model parameters. It is predicted that the topological superconductivity with a higher winding number exhibits a higher value of quantized longitudinal thermal conductance, which is proportional to the winding number [43,59]. Moreover, the accompanying multiple surface Majorana fermions display a new physics of the conformal field theory with a non-Abelian gauge field [59]. Furthermore, owing to a nontrivial spin texture on the Fermi surface originating from the winding number, we can expect nontrivial magnetic responses. We also identify the topological phase diagram of the superconducting state and determine the relevant topological numbers and the patterns of surface Majorana fermions. We finally discuss the experimental signals of high winding superconductivity in the case of $\mathrm{Sr}_{3-x} \mathrm{SnO}$. We observe that $\mathrm{Sr}_{3-x} \mathrm{SnO}$ is in the vicinity of a topological phase transition, which predicts the characteristic nodal structure of the superconducting gap if it realizes the $J=0$ odd-parity pairing state.

This paper is organized as follows. In Sec. II, we examine the normal state of higher-spin topological materials. Using the general $k \cdot p$ Hamiltonian involving two $J=3 / 2$ bands with opposite parity, we obtain the phase diagram of the $J=3 / 2$ topological crystalline insulators. We reproduce the results for the antiperovskite oxides [50-52], clarifying the topological origin of Dirac points in antiperovskites. We also observe that a novel cubic pattern of Dirac points is possible, in addition to the octahedral Dirac points in antiperovskites. In Sec. III, we examine the superconductivity of doped higher-spin TIs. We classify gap functions in terms of an irreducible representation of the $O_{h}$ point group and evaluate the superconducting critical temperature for each gap function. We demonstrate that the $A_{1 u}(J=0)$ representation is the most stable oddparity pairing state, and the $T_{1 u}(J=3$, or 1$)$ is the second one. In Sec. IV, we identify the topological crystalline superconductivity of the $A_{1 u}$ state. Section V discusses the application of our theory to $\mathrm{Sr}_{3-x} \mathrm{SnO}$, the case without band inversion, and the property of other pairing states, and, subsequently, the conclusion is presented in Sec. VI.

\section{NORMAL STATE}

\section{A. Effective Hamiltonian}

Here, we formulate higher-spin TIs. First, we define high-spin fermions. In the presence of spin-orbit coupling, electrons may behave as spin- $J$ fermions with $J=\mid l \pm$ $1 / 2 \mid$ owing to a mixture of the orbital angular momentum $l$ and the electron spin $1 / 2$. For electrons in crystals, however, a more precise definition of $J$ is necessary, as crystals partially break rotation symmetry. Considering that spin $J$ in the above sense indicates $(2 J+1)$-fold degeneracy at $\boldsymbol{k}=0$, we define $\operatorname{spin} J$ in crystals as a 
$(2 J+1)$-dimensional representation of the point group at the $\Gamma$ point. From this definition, it is observed that only a $J=3 / 2$ fermion is possible as a higher-spin fermion, as point groups allow a maximum of four-dimensional representations at $\Gamma$. Furthermore, cubic symmetry is necessary to obtain the four-dimensional representation. Therefore, we consider a cubic crystal in the following analysis. We also assume inversion symmetry for simplicity, which specifies the cubic symmetry as the $O_{h}$ group.

For ordinary spin- $1 / 2$ electrons, a topological insulating phase with inversion symmetry is obtained via band inversion between the orbitals with different parities at the $\Gamma$ point. We can consider a similar band-inversion mechanism even for $J=3 / 2$ electrons. The $O_{h}$ group hosts two different four-dimensional, i.e., $J=3 / 2$, representations, $G_{3 / 2 g}$ and $G_{3 / 2 u}$, corresponding to the even- and oddparity states, respectively. When these bands are inverted, the gap between them closes at $\Gamma$, and, thus, we can expect a nontrivial topological phase transition through the band inversion.

This argument specifies a system for a higher-spin TI. It is symmetric under the $O_{h}$ group and consists of two different $J=3 / 2$ bands corresponding to the $G_{3 / 2 g}$ and $G_{3 / 2 u}$ representations of the $O_{h}$ group. Moreover, timereversal symmetry should be assumed when we consider an insulator.

The single-particle state of the $J=3 / 2$ TI is represented by an eight-component spinor $\boldsymbol{c}_{\boldsymbol{k}}$ composed of the annihilation operator $c_{j_{z}, \sigma_{z}, k}$, where $j_{z}= \pm 3 / 2, \pm 1 / 2$ denotes the $z$ component of $J$ corresponding to the four-dimensional representation of the $O_{h}$ group, $\sigma_{z}$ specifies the parity of the representation, i.e., $\sigma_{z}=1\left(\sigma_{z}=-1\right)$ for the $G_{3 / 2 g}\left(G_{3 / 2 u}\right)$ representation with even (odd) parity, which can be characterized as $d, g,(p, f)$, or higher-order orbitals, and $\boldsymbol{k}$ is the momentum. Generators of the $O_{h}$ group consist of $q$-fold discrete crystal rotations $C_{q, n}(q=2,3,4)$ and inversion $P$, which act on the Hamiltonian $H_{0}(\boldsymbol{k})$ as, respectively,

$$
C_{q, \boldsymbol{n}} H_{0}(\boldsymbol{k}) C_{q, \boldsymbol{n}}^{-1}=H_{0}\left(D_{C_{q, n}}[\boldsymbol{k}]\right) \quad \text { with } \quad C_{q, \boldsymbol{n}}=e^{-i(2 \pi / q) \boldsymbol{J} \cdot \boldsymbol{n}},
$$

$$
P H_{0}(\boldsymbol{k}) P^{-1}=H_{0}(-\boldsymbol{k}) \quad \text { with } \quad P=\sigma_{z} .
$$

Here, $\boldsymbol{n}$ denotes the rotation axis, $D_{C_{q, n}}[\boldsymbol{k}]$ indicates the $q$-fold rotation of $\boldsymbol{k}, J_{i=x, y, z}$ is the $4 \times 4 J=3 / 2$ spin operator (see Appendix A), and $\sigma_{i}$ is the $2 \times 2$ Pauli matrix in the orbital space. As the $G_{3 / 2 u}\left(G_{3 / 2 g}\right)$ state changes (does not change) the sign under inversion, $P$ is given as $P=\sigma_{z}$. We also have time-reversal symmetry,

$$
\mathcal{T} H_{0}(\boldsymbol{k}) \mathcal{T}^{-1}=H_{0}(-\boldsymbol{k}) \quad \text { with } \quad \mathcal{T}=C_{2, y} \mathcal{K},
$$

with the complex conjugate operator $\mathcal{K}$.
In the following analysis, we use the low-energy effective Hamiltonian for the $J=3 / 2$ electrons. To construct the effective Hamiltonian, we consider possible scalar matrices with respect to $O_{h}$ symmetry. First, we note that $\sigma_{z}$ and $\sigma_{0}$ are scalars, whereas $\sigma_{x}$ and $\sigma_{y}$ are pseudoscalars. Any $\sigma_{\nu}$ $(\nu=0, x, y, z)$ is invariant under rotation, but $\sigma_{x}$ and $\sigma_{y}$ change their signs under inversion. Second, as the spin operators $J_{i}(i=x, y, z)$ and their third-order polynomials $\tilde{J}_{i} \equiv \frac{5}{3} \sum_{j \neq i} J_{j} J_{i} J_{j}-\frac{7}{6} J_{i}$ (see also Appendix A) behave as pseudovectors for the $O_{h}$ point group, the inner product $\boldsymbol{k} \cdot \boldsymbol{J}$ and $\boldsymbol{k} \cdot \tilde{\boldsymbol{J}}$ are pseudoscalars [60]. Finally, we can construct a scalar as a product of any two of the above pseudoscalars. Consequently, the effective Hamiltonian for the lower order of momentum $\boldsymbol{k}$ is given as [52]

$$
H_{0}(\boldsymbol{k})=m(\boldsymbol{k}) \sigma_{z}+\boldsymbol{k} \cdot\left(v_{1} \boldsymbol{J}+v_{2} \tilde{\boldsymbol{J}}\right) \sigma_{x}-\mu \sigma_{0},
$$

where the $\sigma_{y}$ term is absent owing to the time-reversal symmetry.

The effective mass $m(\boldsymbol{k})$ in Eq. (4) is given as

$$
m(\boldsymbol{k})=-m_{0}+\alpha k^{2}+\left[\boldsymbol{k} \cdot\left(\beta_{1} \boldsymbol{J}+\beta_{2} \tilde{\boldsymbol{J}}\right)\right]\left[\boldsymbol{k} \cdot\left(\beta_{3} \boldsymbol{J}+\beta_{4} \tilde{\boldsymbol{J}}\right)\right],
$$

up to the second order of $\boldsymbol{k}$, where $2 m_{0}$ is the energy gap between the $G_{3 / 2 u}$ and $G_{3 / 2 g}$ states at the $\Gamma$ point. In the following analysis, we focus on the case where band inversion occurs at the $\Gamma$ point. We consider $m_{0}>0$, $\alpha>0$, and $\beta_{i}=0(i=1,2,3,4)$, which can reproduce the band structure of $\mathrm{Sr}_{3} \mathrm{SnO}$ qualitatively. The case without band inversion is briefly discussed in Sec. V B.

The second term of Eq. (4) with $\sigma_{x}$ leads to orbital mixing between even- and odd-parity states, which is represented in green in Figs. 1(a) and 1(b). This mixture opens the global energy gap around $E=-\mu$ except for the parameter points displayed in Fig. 1. Note that the $\tilde{\boldsymbol{J}}$ term reduces the continuous rotational symmetry to the discrete $O_{h}$ one.

The $\sigma_{0}$ term in Eq. (4) provides the Fermi level. In this section, to demonstrate the topological property of the band gap discussed above, we set $\mu=0$, where the Fermi level lies in the band gap and the system falls into the insulating state. The large $|\mu|$ pushes the energy gap below or above the Fermi level. In this case, the system is a metal rather than an insulator, although the topological property of the band gap remains unchanged. This metallic state is important to induce topological superconductivity, which is discussed later.

Note that $H_{0}(\boldsymbol{k})$ cannot be an ordinary TI, although band inversion can occur at the $\Gamma$ point. The $\boldsymbol{Z}_{2}$ number for TI is always trivial owing to the fourfold degeneracy of the $J=3 / 2$ spin at $\Gamma$. However, the system can be a topological crystalline insulator, as shown below. 


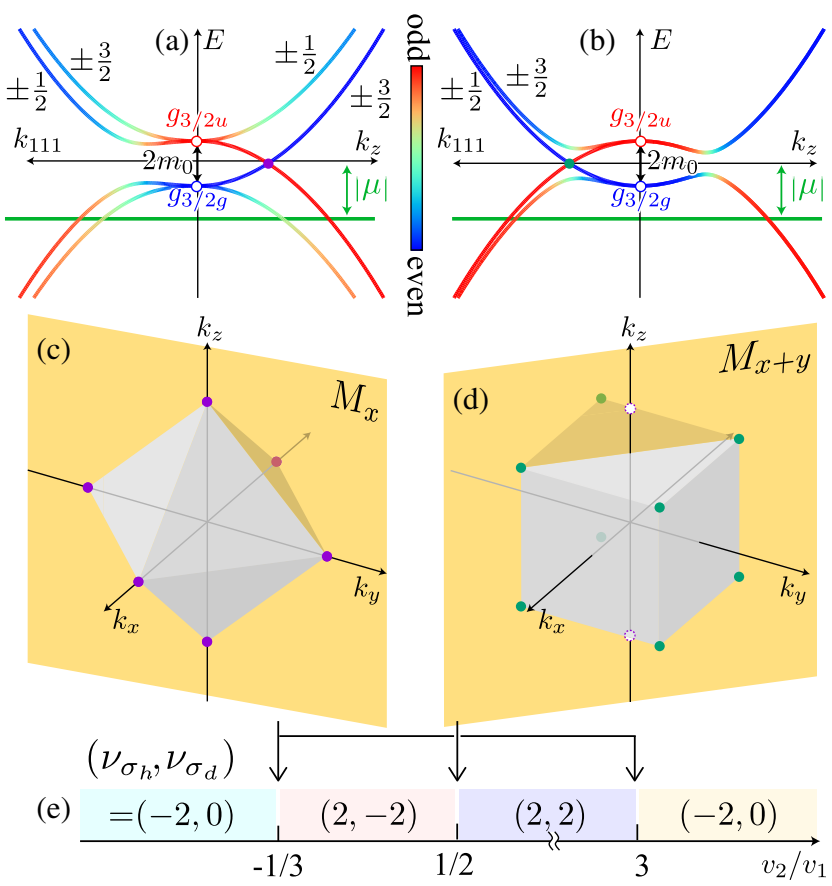

FIG. 1. Energy dispersion near the $\Gamma$ point for (a) $v_{2}=3 v_{1}$ and (b) $v_{2}=v_{1} / 2$. The dispersions along $k_{z}$ and $k_{111}=\left(k_{x}+k_{y}+\right.$ $\left.k_{z}\right) / \sqrt{3}$ are labeled by the $z$ and (111) component of angular momenta $j_{z}$ and $j_{111}$, respectively. For the hole-doped antiperovskite material $\mathrm{Sr}_{3-x} \mathrm{SnO}$, the Fermi level is located below the fourfold degeneracy point $E=-m_{0}$ as indicated by the green line. (c) and (d) show the octahedral and cubic Dirac points for $v_{2}=3 v_{1}$ and $v_{2}=v_{1} / 2$, respectively. The dots denote the Dirac points. The transparent panels indicate the mirror plane defining the topological phase transition with the creation of the Dirac points. (e) shows the horizontal and diagonal mirror Chern numbers $\nu_{\sigma_{h}}$ and $\nu_{\sigma_{d}}$ as a function of $v_{2} / v_{1}$.

\section{B. Octahedral Dirac points}

By diagonalizing the Hamiltonian (4), we obtain the band spectrum. First, let us focus on the band spectrum along the primary axis, say, the $k_{z}$ axis. On the $k_{z}$ axis, we have $\left[C_{4, z}, H_{0}\left(0,0, k_{z}\right)\right]=0$ owing to the fourfold rotation symmetry of the $O_{h}$ group, $C_{4, z} H_{0}\left(-k_{y}, k_{x}, k_{z}\right) C_{4, z}^{-1}=$ $H_{0}(\boldsymbol{k})$, and, thus, the Hamiltonian is subdivided into the eigensector of $C_{4, z}$ with the eigenvalue $\lambda_{C_{4, z}}=e^{-i\left(j_{z} / 2\right) \pi}$ :

$$
H_{0, j_{z}}\left(k_{z}\right)=m_{j_{z}}\left(k_{z}\right) \sigma_{z}+b_{j_{z}} k_{z} \sigma_{x},
$$

where $m_{j_{z}}\left(k_{z}\right)=m_{-j_{z}}\left(k_{z}\right)$ is an even function of $k_{z}$ and $b_{j_{z}}$ is the following constant:

$$
\begin{aligned}
& b_{ \pm 3 / 2}= \pm\left(3 v_{1}-v_{2}\right) / 2 \\
& b_{ \pm 1 / 2}= \pm\left(v_{1}+3 v_{2}\right) / 2 .
\end{aligned}
$$

From time-reversal and inversion symmetry, the sectors with opposite $j_{z}$ form a Kramers pair with the same spectra.
The first term in Eq. (5) provides a parabolic energy dispersion for each orbital, and the second one mixes them. As shown in Eq. (6) [Eq. (7)], the orbital mixing in the $j_{z}= \pm 3 / 2\left(j_{z}= \pm 1 / 2\right)$ sector vanishes when $v_{2}=3 v_{1}$ $\left(v_{1}=-3 v_{2}\right)$. In such cases, Dirac points appear in the band spectrum.

Figure 1(a) illustrates the band spectra for $v_{2}=3 v_{1}$. The two parabolic dispersions with opposite orbital parity in the spin $j_{z}= \pm 3 / 2$ sector linearly cross each other at $k_{z}=$ $\pm k_{0}$ with $k_{0}$ satisfying $m_{ \pm 3 / 2}\left(k_{0}\right)=0$. Consequently, there appear two Dirac points on the $k_{z}$ axis. From the $O_{h}$-group symmetry, their counterparts also exist on the $k_{x}$ and $k_{y}$ axes, respectively, forming vertices of an octahedron in the momentum space. See Fig. 1(c). Furthermore, orbitals with opposite parity in the $j_{z}= \pm 1 / 2$ sectors hybridize with each other, and, thus, they do not form a Dirac point. Similarly, when $v_{2}=-v_{1} / 3$, octahedral Dirac points appear in the $j_{z}= \pm 1 / 2$ sectors.

In contrast to ordinary Dirac semimetals such as $\mathrm{Cd}_{3} \mathrm{As}_{2}$, these Dirac points are easily gapped out by a small perturbation even if crystalline symmetry is preserved. Thus, they are not stable, but their existence is not accidental.

They indeed appear as gap-closing points accompanied by a topological phase transition. A key topological number is the mirror Chern number. Consider one of three equivalent horizontal mirror reflections of the $O_{h}$ group, say, the mirror reflection with respect to the $y z$ plane:

$$
M_{x} H_{0}\left(-k_{x}, k_{y}, k_{z}\right) M_{x}^{-1}=H_{0}(\boldsymbol{k}),
$$

with $M_{x}=P C_{2, x}$. See Fig. 1(c). On the mirror-invariant $k_{x}=0$ plane, it holds that $\left[M_{x}, H_{0}\left(0, k_{y}, k_{z}\right)\right]=0$, and, hence, the Hamiltonian has the block-diagonal form $H_{0}\left(0, k_{y}, k_{z}\right)=\operatorname{diag}\left[H_{0}^{+i}\left(0, k_{y}, k_{z}\right), H_{0}^{-i}\left(0, k_{y}, k_{z}\right)\right]$ in the eigenbasis of $M_{x}$ with the eigenvalues $\lambda_{M_{x}}= \pm i$. Each sector may host its own Chern number

$$
\nu_{\mathrm{Ch}}^{ \pm i}=-i \sum_{\xi_{n}^{ \pm i}<0} \int_{k_{x}=0} d^{2} k \epsilon^{\alpha \beta}\left\langle\partial_{\alpha} u_{n, k}^{ \pm i} \mid \partial_{\beta} u_{n, \boldsymbol{k}}^{ \pm i}\right\rangle,
$$

where we use the solution of the Bloch equation $H_{0}^{ \pm i}\left|u_{n, \boldsymbol{k}}^{ \pm i}\right\rangle=\xi_{n}^{ \pm i}\left|u_{n, \boldsymbol{k}}^{ \pm i}\right\rangle$ and two-dimensional Levi-Civita symbol $\epsilon^{\alpha \beta}$ with $\alpha, \beta \in\left\{k_{y}, k_{z}\right\}$. The mirror Chern number $\nu_{\sigma_{h}}$ for the horizontal mirror reflection is defined by $\nu_{\sigma_{h}}=\left(\nu_{\mathrm{Ch}}^{+i}-\nu_{\mathrm{Ch}}^{-i}\right) / 2$. From time-reversal symmetry, the total Chern number $\nu_{\mathrm{Ch}}^{+i}+\nu_{\mathrm{Ch}}^{-i}$ vanishes, and, thus, $\nu_{\sigma_{h}}$ is an integer.

As summarized in Fig. 1(e), the mirror Chern number in the present system is obtained as [52]

$$
\nu_{\sigma_{h}}=2 \operatorname{sgn}\left(b_{3 / 2} b_{1 / 2}\right)
$$

which jumps by four when Dirac points appear at $v_{2}=3 v_{1}$ or $v_{2}=-v_{1} / 3$. In other words, each of the four Dirac 
points on the $k_{x}=0$ plane change the mirror Chern number by one. Therefore, these Dirac points are caused by the topological phase transition, which indicates that the Dirac points must appear in the phase diagram because they separate two distinct topological phases, even though for any specific choice of parameters (i.e., one point in the phase diagram) they can gapped. The small perturbation, such as mixing from other bands not considered in the lowenergy effective Hamiltonian (4), may open a gap in the Dirac points, but it merely shifts the phase boundary of the topological phase transition, which also hosts gapless Dirac points. Except at the topological phase transition, the system supports a nonzero mirror Chern number, and, thus, the normal state is a topological crystalline insulator if the Fermi level is in the band gap.

\section{Cubic Dirac points}

The effective Hamiltonian (4) also reveals that another class of Dirac points is possible in higher-spin topological materials. To demonstrate this possibility, consider the diagonal axis, say, the (111) axis. From the threefold rotational symmetry of the $O_{h}$ group, the Hamiltonian along the (111) axis in momentum space is subdivided into eigensectors of $C_{3,(111)}$ with the eigenvalues $\lambda_{C_{3,(11)}}=$ $e^{-i(2 / 3) j_{111} \pi}$, where $(111)=(\hat{\boldsymbol{x}}+\hat{\boldsymbol{y}}+\hat{\boldsymbol{z}}) / \sqrt{3}$ and $j_{111}=$ $\pm 1 / 2, \pm 3 / 2$. Note that $j_{111}= \pm 3 / 2$ falls into the same eigensector with $\lambda_{C_{3,(11)}}=-1$. The Hamiltonian in the subsector takes the same form as Eq. (5), but $k_{z}, j_{z}$, and $b_{j_{z}}$ in Eq. (5) are replaced with $k_{111}=\left(k_{x}+k_{y}+k_{z}\right) / \sqrt{3}$, $j_{111}$, and $b_{j_{111}}^{\prime}$, respectively. The interorbital coupling $b_{j_{111}}^{\prime}$ is given by

$$
\begin{gathered}
b_{ \pm 1 / 2}^{\prime}= \pm \frac{v_{1}-2 v_{2}}{2} \\
b_{ \pm 3 / 2}^{\prime}=\left[\left(\frac{3}{2} v_{1}+\frac{1}{3} v_{2}\right) \eta_{z}+\frac{5 \sqrt{2}}{6} v_{2} \eta_{x}\right] .
\end{gathered}
$$

Here, $\eta_{i}$ is the Pauli matrix in the $j_{111}= \pm 3 / 2$ space.

$b_{ \pm 3 / 2}^{\prime}$ does not vanish for any $v_{i}$, whereas $b_{ \pm 1 / 2}^{\prime}$ becomes zero when $v_{2}=v_{1} / 2$. In the latter case, the orbitals with opposite parity do not hybridize with each other along the (111) axis, forming a Dirac point in Fig. 1(b). By considering $O_{h}$ symmetry, we have a total of eight Dirac points at the vertices of a cube, as illustrated in Fig. 1(d).

The cubic Dirac points are also understood as gapclosing points for the topological phase transition. The relevant topological index in the present case is the mirror Chern number for the diagonal mirror reflection of the $O_{h}$ group. For instance, consider the diagonal mirror reflection with respect to the $k_{x}+k_{y}=0$ plane in Fig. 1(d). Similar to the octahedral Dirac case, we can evaluate the mirror Chern number $\nu_{\sigma_{d}}$ with respect to the diagonal mirror plane. The result is shown in Fig. 1(f). At $v_{2}=v_{1} / 2$, where there are four Dirac points on this plane, the mirror Chern number jumps by four.

Notably, two of the octahedral Dirac points appear on the diagonal mirror plane when $v_{2}=3 v_{1}$ or $v_{2}=-v_{1} / 3$. Consequently, the diagonal mirror Chern number in Fig. 1(f) jumps by two at $v_{2}=3 v_{1}$ and $v_{2}=-v_{1} / 3$.

\section{SUPERCONDUCTIVITY}

\section{A. $O_{h}$ classification of the gap function}

With carrier doping, TIs can be superconductors at a low temperature. Here, we consider the superconducting states of doped higher-spin TIs.

To describe the superconducting states, we use the Nambu space spanned by the basis $\left(\boldsymbol{c}_{\boldsymbol{k}}, \overline{\boldsymbol{c}}_{-\boldsymbol{k}}\right)$ with the eight-component spinor $\boldsymbol{c}_{\boldsymbol{k}}$ of the annihilation operator and its time-reversal hole partner $\overline{\boldsymbol{c}}_{-\boldsymbol{k}}$ with the component $\bar{c}_{j_{z}, \sigma_{z},-\boldsymbol{k}}=\sum_{j_{z}^{\prime}}\left(C_{2, y}\right)_{j_{z}, j_{z}^{\prime}} c_{j_{z}^{\prime}, \sigma_{z},-\boldsymbol{k}}^{\dagger}$. In this basis, the BdG Hamiltonian is written as

$$
H(\boldsymbol{k})=H_{0}(\boldsymbol{k}) \tau_{z}+\operatorname{Re}[\Delta(\boldsymbol{k})] \tau_{x}+\operatorname{Im}[\Delta(\boldsymbol{k})] \tau_{y},
$$

where $\tau_{\mu}$ represents the $2 \times 2$ matrices acting on the particle and time-reversal hole space and we use time-reversal symmetry for the one-particle Hamiltonian $\mathcal{T} H_{0}(\boldsymbol{k}) \mathcal{T}^{-1}=$ $H_{0}(-\boldsymbol{k})$. Note that, in this basis, the point group and timereversal act on the gap function $\Delta(\boldsymbol{k})$ in the same manner as Eqs. (1)-(3) on the normal Hamiltonian $H_{0}(\boldsymbol{k})$ (see Appendix B for more details).

We classify the multicomponent gap function $\Delta(\boldsymbol{k})$. For simplicity, we assume that $\Delta(\boldsymbol{k})$ is independent of the momentum $\boldsymbol{k}$ as in the conventional BCS theory. The higher-spin and multiorbital natures of the system enable us to consider various unconventional Cooper pairs even in the weakly correlated case.

The gap function is expanded as

$$
\Delta=\sum_{i, \nu} \Delta_{i, \nu} \Phi_{i, \nu}, \quad \Phi_{i, \nu}=\frac{\varphi_{i}(\boldsymbol{J}) \otimes \sigma_{\nu}}{N},
$$

where $\varphi_{i}(\boldsymbol{J})$ is a set of $4 \times 4$ matrices spanning the spin space, $\sigma_{\nu}(\nu=0, x, y, z)$ is the Pauli matrix in the orbital space, and $N=\sqrt{\operatorname{tr}\left[\left(\varphi_{i} \otimes \sigma_{\nu}\right)^{2}\right] / 8}$ is the normalization constant. A convenient basis of $\varphi_{i}(\boldsymbol{J})$ is the spherical harmonic $Y_{l, m}(\boldsymbol{J})$, which is defined by $Y_{l, m \pm 1}=$ $\mp\left[J_{ \pm}, Y_{l, m}\right]$ with $J_{ \pm}=J_{x} \pm i J_{y}$. In the present case, the spin of a Cooper pair is given by $3 / 2 \otimes 3 / 2=$ $0 \oplus 1 \oplus 2 \oplus 3$, and, hence, the azimuthal and magnetic quantum numbers $l$ and $m$ in $Y_{l, m}$ assume the values $l=0$, $1,2,3$ and $m=-l, \ldots, l$, respectively. Reconstructing the basis $Y_{l, m}$ for each $l$ in terms of the representations of the $O$ group, we obtain $\varphi_{i}(\boldsymbol{J})$ in Table I. Here, we use the $O$ group rather than $O_{h}$, as inversion acts on the spin space trivially as the identity operator. 
TABLE I. Classification of the spin basis for the $4 \times 4$ matrices according to $O$-point-group symmetry. The columns correspond to an irreducible representation of $O$-point-group symmetry, with indices for the amplitude $l$ of coupled angular momentum for two $3 / 2$ spins, and the basis matrix.

\begin{tabular}{ll}
\hline \hline$O^{(l)}$ & \multicolumn{1}{c}{ Basis $\varphi_{i}(\boldsymbol{J})$} \\
\hline$A_{1}^{(0)}$ & 1 \\
$T_{1}^{(1)}$ & $\left\{J_{x}, J_{y}, J_{z}\right\}$ \\
$E^{(2)}$ & $\left\{2 J_{z}^{2}-J_{x}^{2}-J_{y}^{2}, J_{x}^{2}-J_{y}^{2}\right\}$ \\
$T_{2}^{(2)}$ & $\left\{J_{x} J_{y}+J_{y} J_{x}, J_{y} J_{z}+J_{z} J_{y}, J_{z} J_{x}+J_{x} J_{z}\right\}$ \\
$A_{2}^{(3)}$ & $J_{x} J_{y} J_{z}+J_{z} J_{y} J_{x}$ \\
$T_{1}^{(3)}$ & $\left\{\tilde{J}_{x}, \tilde{J}_{y}, \tilde{J}_{z}\right\}$ \\
$T_{2}^{(3)}$ & $\left\{J_{y} J_{x} J_{y}-J_{z} J_{x} J_{z}, J_{z} J_{y} J_{z}-J_{x} J_{y} J_{x}, J_{x} J_{z} J_{x}-J_{y} J_{z} J_{y}\right\}$ \\
\hline \hline
\end{tabular}

Notably, a possible combination of $\varphi_{i}(\boldsymbol{J})$ and $\sigma_{\nu}$ in the right-hand side of Eq. (14) is restricted by the constraint

$$
\left[\Delta(\boldsymbol{k}) C_{2, \boldsymbol{y}}\right]^{T}=-\Delta(-\boldsymbol{k}) C_{2, \boldsymbol{y}}
$$

from the Fermi statistics of $\boldsymbol{c}_{\boldsymbol{k}}$. As $\varphi_{i}(\boldsymbol{J})$ for even (odd) $l$ satisfies $\quad\left[\varphi_{i}(\boldsymbol{J}) C_{2, y}\right]^{T}=-\varphi_{i}(\boldsymbol{J}) C_{2, \boldsymbol{y}} \quad\left(\left[\varphi_{i}(\boldsymbol{J}) C_{2, \boldsymbol{y}}\right]^{T}=\right.$ $\left.+\varphi_{i}(J) C_{2, y}\right)$, it is combined only with the symmetric Pauli matrices $\sigma_{0}, \sigma_{x}$, and $\sigma_{z}$ (the antisymmetric Pauli matrix $\sigma_{y}$ ). Consequently, we have 28 different $\Phi_{i, \nu}$ in Eq. (14). In the group theory, they are classified into eight irreducible representations of the $O_{h}$ group, as shown in Table II.

Here, we compare the possible gap functions in Table II with those in the superconductor in the single $J=3 / 2$ band. In single $J=3 / 2$ systems such as half-Heusler systems, only even-parity superconductivity corresponding to $A_{1 g}, E_{g}$, and $T_{2 g}$ in Table II is possible within the

TABLE II. Eight classes of the whole basis of the gap function according to $O_{h}$-point-group symmetry. The columns denote intraorbital or interorbital pairing, irreducible representation of $O_{h}$-point-group symmetry, and the basis matrix given as the direct product of spin basis in $O$ representation and Pauli matrices for the orbital basis.

\begin{tabular}{lccc}
\hline \hline Orbital & Parity & $O_{h}$ & Basis $\varphi_{i}(\boldsymbol{J}) \otimes \sigma_{\nu}$ \\
\hline \multirow{4}{*}{ Intra } & & $A_{1 g}$ & $A_{1}^{(0)} \otimes \sigma_{0}, A_{1}^{(0)} \otimes \sigma_{z}$ \\
& Even & $E_{g}$ & $E^{(2)} \otimes \sigma_{0}, E^{(2)} \otimes \sigma_{z}$ \\
& & $T_{2 g}$ & $T_{2}^{(2)} \otimes \sigma_{0}, T_{2}^{(2)} \otimes \sigma_{z}$ \\
\hline \multirow{4}{*}{ Inter } & $A_{1 u}$ & $A_{1}^{(0)} \otimes \sigma_{x}$ \\
& & $A_{2 u}$ & $A_{2}^{(3)} \otimes \sigma_{y}$ \\
& & $E_{u}$ & $E^{(2)} \otimes \sigma_{x}$ \\
& & $T_{1 u}$ & $T_{1}^{(1)} \otimes \sigma_{y}, T_{1}^{(3)} \otimes \sigma_{y}$ \\
& & $T_{2 u}$ & $T_{2}^{(2)} \otimes \sigma_{x}, T_{2}^{(3)} \otimes \sigma_{y}$ \\
\hline \hline
\end{tabular}

$\boldsymbol{k}$-independent gap function [38], because they do not have d.o.f. of $\sigma_{\nu}$ associated with orbital parity. In addition, we have odd-parity superconductivity in Table II. These oddparity pairing states are expected to be topologically nontrivial $[17,19]$. In the present system, these superconductivities are realized with a constant gap function in contrast to the $k$-dependent ones in single $J=3 / 2$ states [41].

\section{B. Critical temperatures}

Now, we evaluate the superconducting transition temperature $T_{c}$ for each representation in Table II. Here, we assume the following density-density pairing interactions:

$$
H_{\mathrm{int}}=-\int d^{3} r\left[U\left(n_{+}^{2}+n_{-}^{2}\right)+2 V n_{+} n_{-}\right] \text {, }
$$

where $n_{\sigma_{z}}(\boldsymbol{r})=\sum_{j_{z}} \psi_{j_{z}, \sigma_{z}}^{\dagger}(\boldsymbol{r}) \psi_{j_{z}, \sigma_{z}}(\boldsymbol{r})$ is the density of the electron with orbital $\sigma_{z}$ defined by the field operator $\psi_{j_{z}, \sigma_{z}}(\boldsymbol{r})=\int d^{3} k e^{-i \boldsymbol{k} \cdot \boldsymbol{r}} c_{j_{z}, \sigma_{z}, \boldsymbol{k}}$.

The transition temperature $T_{c}$ is obtained by solving the linearized gap equation. For each representation in Table II, we have $[18,36]$ (see also Appendix B)

$$
\operatorname{det} X\left(T_{c}\right)=0 \text {, }
$$

where the matrix element of $X\left(T_{c}\right)$ is

$$
\begin{aligned}
X_{\alpha \alpha^{\prime}}\left(T_{c}\right) \sim & \delta_{\alpha \alpha^{\prime}}+\sum_{n} \frac{I\left(T_{c}\right)}{2} \int_{k_{F}} d^{2} k^{\prime} D_{n}\left(\boldsymbol{k}^{\prime}\right) V_{\alpha} \\
& \times \sum_{n^{\prime}=n, \bar{n}}\left\langle u_{n^{\prime}, \boldsymbol{k}^{\prime}}\left|\Phi_{\alpha}\right| u_{n, \boldsymbol{k}^{\prime}}\right\rangle\left\langle u_{n, \boldsymbol{k}^{\prime}}\left|\Phi_{\alpha^{\prime}}\right| u_{n^{\prime}, \boldsymbol{k}^{\prime}}\right\rangle .
\end{aligned}
$$

Here, $\left|u_{n, k}\right\rangle$ is the normalized single-particle state for the Fermi surface of the $n$th band defined by $H_{0}(\boldsymbol{k})\left|u_{n \boldsymbol{k}}\right\rangle=0$. (Hereafter, all the ket vectors $\left|u_{n, k}\right\rangle$ indicate the wave function of the normal state unless otherwise noted.) The band index $\bar{n}$ represents the state $\left|u_{\bar{n} k}\right\rangle=P \mathcal{T}\left|u_{n, k}\right\rangle$, which is degenerated with $\left|u_{n \boldsymbol{k}}\right\rangle . D_{n}\left(\boldsymbol{k}^{\prime}\right)=\left[\left(d k_{F}\right) /\left(d \xi_{n}\right)\right]$ is the density of states on the Fermi surface. The interaction is $V_{\alpha}=U(V)$ for intraorbital (interorbital) pairing. We also use $I\left(T_{c}\right)=\int_{-\hbar \omega_{0}}^{\hbar \omega_{0}} d \xi[1 /(2 \xi)] \tanh \left[\xi /\left(2 k_{B} T_{c}\right)\right] \sim \ln \left[\left(2 e^{\gamma} \hbar \omega_{0}\right) /\right.$ $\left(\pi k_{B} T_{c}\right)$ ], Euler's constant $\gamma$, cutoff frequency $\omega_{0}$, and Boltzmann constant $k_{B}$. Here, $\alpha$ and $\alpha^{\prime}$ specify a different basis $\varphi_{i}(\boldsymbol{J}) \otimes \sigma_{\nu} / N \equiv \Phi_{\alpha^{\prime}}$ (if existing) in the representation. For instance, the $A_{1 g}$ gap has two different bases, $A_{1}^{(0)} \otimes \sigma_{0}$ and $A_{1}^{(0)} \otimes \sigma_{z}$, and, hence, $\alpha^{\prime}$ runs $\alpha^{\prime}=1,2$.

We show the numerical solutions of Eq. (17) in Fig. 2. The results for even-parity states and those for odd ones are shown separately as $T_{c}$ 's of even-parity (odd-parity) states are independent of the interorbital (intraorbital) pairing interaction $V(U)$. It is observed that the spin-singlet $A_{1 g}$ 




FIG. 2. Transition temperature for (a) even- and (b) oddparity superconducting states. The unit temperature is $T_{0}=$ $\left[\left(2 e^{\gamma} \hbar \omega_{0}\right) /\left(\pi k_{B}\right)\right]$ with Euler's constant $\gamma$ and cutoff frequency $\omega_{0}$. Here, the parameters are $\mu=-2 m_{0}, \alpha=6.25 v_{1}^{2} / m_{0}$, and $\beta_{i}=0$.

$\left(A_{1 u}\right)$ representation exhibits the highest $T_{c}$ among evenparity (odd-parity) states.

These results can be understood using the spin and orbital textures of the Fermi surfaces in the normal state. In Fig. 3, we show the expectation values of $\langle\boldsymbol{J}\rangle$ and $\langle\boldsymbol{\sigma}\rangle$ on the Fermi surfaces with respect to the single-particle state $\left|u_{n k}\right\rangle$. The hole-doped system with $\mu<-m_{0}$ supports two Fermi surfaces around the $\Gamma$ point, and each Fermi surface has twofold degeneracy with different spin and orbital textures, owing to the coexistence of inversion and time-reversal symmetries (see also Appendix B). The orbital texture $\langle\boldsymbol{\sigma}\rangle$ indicates mixing between orbitals with even and odd parity. When $\langle\boldsymbol{\sigma}\rangle \| \hat{z}$, the corresponding single-particle state satisfies $\sigma_{z}= \pm 1$, and, hence, it should be an orbital with either even or odd parity and there is no orbital mixing. In contrast, when $\langle\boldsymbol{\sigma}\rangle \| \hat{x}$, orbitals with even and odd parity are fully mixed in equal weight.

First, consider the $A_{1 g}$ state. As it is a spin singlet with the angular momentum $J=0$, the Cooper pair has an antiparallel spin configuration. Thus, it is formed by an electron and its time-reversal partner, which have opposite spins. In this case, they can form an intraorbital pairing without obstruction. Indeed, as shown in Fig. 3, as time reversal does not change the orbital, the electron and the partner have the same $\langle\boldsymbol{\sigma}\rangle$. Consequently, the $A_{1 g}$ state is naturally realized in the presence of the intraorbital pairing interaction. It corresponds to the usual $s$-wave superconductivity, where the bulk is topologically trivial (see also Sec. V C).

Similarly, the $A_{1 u}$ state is naturally realized in the presence of the interorbital pairing interaction. The $A_{1 u}$ state is also spin singlet with $J=0$, and, hence, its Cooper pair is also formed between an electron and its time-reversal partner. However, for the interorbital pairing state, orbital mixing is necessary, which results in an interesting nodal

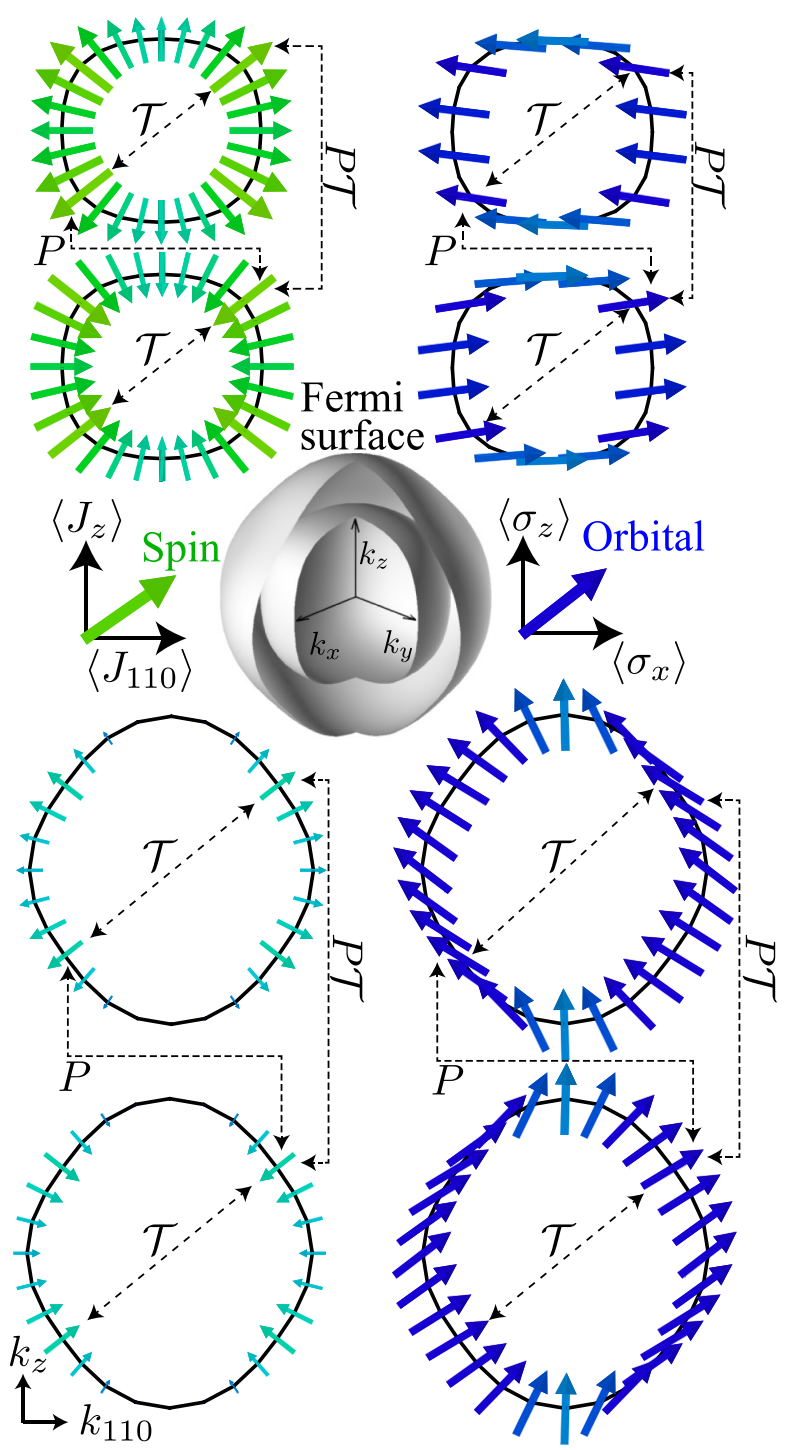

FIG. 3. (Center) Fermi surfaces of antiperovskites. For $\mu<-m_{0}$, there are two Fermi surfaces. The top and bottom panels show the spin and orbital textures of the inner and outer Fermi surfaces, respectively. The green (blue) arrow represents the expectation value of the spin (orbit). The dashed arrows indicate $\mathcal{T}, P$, and $P \mathcal{T}$ partners. The parameters are $\mu=-2 m_{0}$, $v_{2}=2.9 v_{1}, \alpha=6.25 v_{1}^{2} / m_{0}, \beta_{i}=0$, and $m_{0}>0$.

structure of the gap depending on the model parameters. For instance, when $v_{2}=3 v_{1}$, the outer Fermi surface does not show orbital mixing at the intersections with the primary axis (i.e., the $k_{i=x, y, z}$ axis). See Fig. 3. Correspondingly, there are gap nodes at the intersections in Fig. 4(b). We show later that this nodal structure is not accidental but has a topological origin. Note that, except for the special point $v_{2}=3 v_{1}, v_{1} / 2$, and $-v_{1} / 3$, the $A_{1 u}$ state is full gap as shown in Fig. 4(c). In Fig. 5, we show the phase diagram obtained through our calculation. It is observed that the $A_{1 u}$ phase is realized when $V$ is much larger than $U$. 


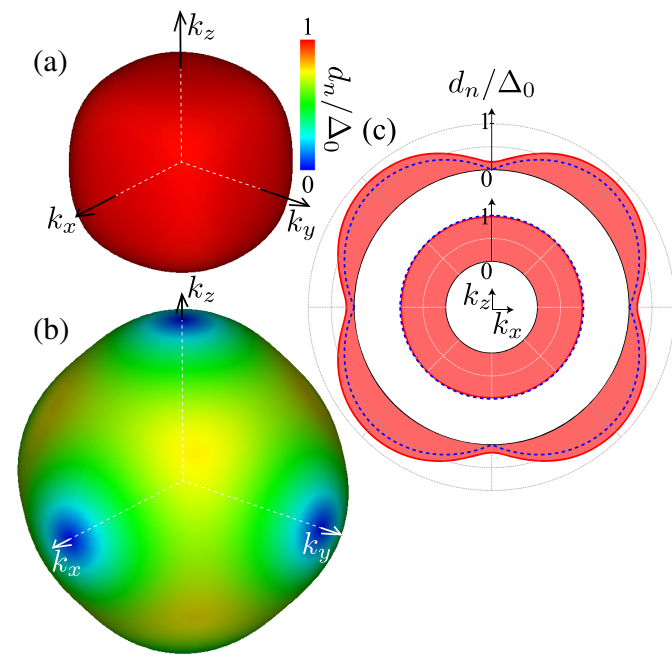

FIG. 4. Energy gap $d_{n}=\left\langle u_{n, k}|\Delta| u_{n, k}\right\rangle$ of $A_{1 u}$ representation at the inner (a) and outer (b) Fermi surfaces for $v_{2}=3 v_{1}$. Those for $v_{2}=3 v_{1}$ (dashed line) and $4 v_{1}$ (solid line with filled lobe) on the $k_{z} k_{x}$ cut are displayed in (c).

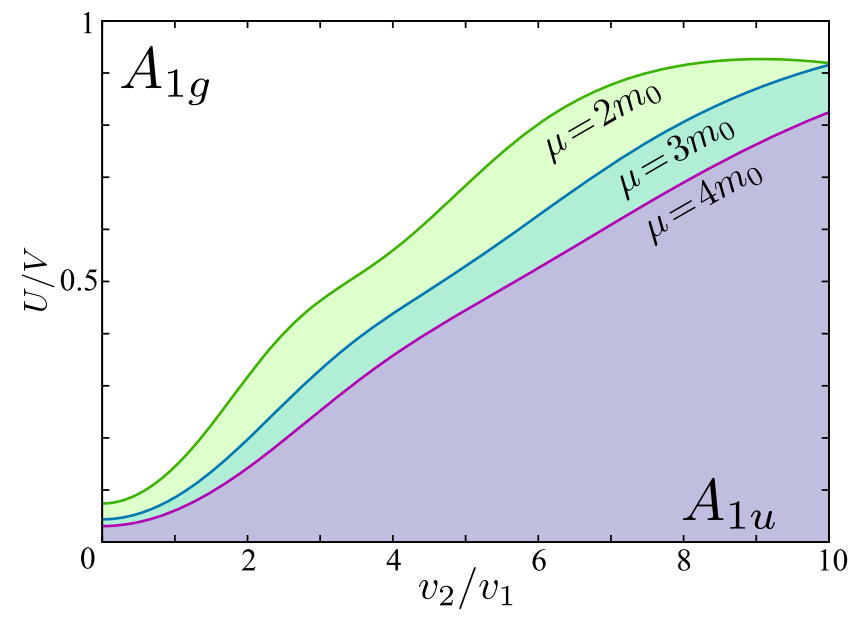

FIG. 5. Phase diagram of odd- and even-parity states spanned by $U / V$ and $v_{2} / v_{1}$. The curves are the phase boundary for the typical values of the chemical potential. The other parameters are the same as in Fig. 3.

\section{TOPOLOGICAL SUPERCONDUCTIVITY IN THE $A_{1 u}$ STATE}

In this section, we study the topological properties of the $A_{1 u}(J=0)$ superconducting state, in terms of the topological indices and numerically obtained surface states.

\section{A. 3D winding number}

We first examine the topological properties independent of the crystal symmetry. For the time-reversal symmetric superconductivity generally described by the BdG Hamiltonian (13), we have chiral symmetry:

$$
\Gamma H(\boldsymbol{k}) \Gamma=-H(\boldsymbol{k}) \quad \text { with } \quad \Gamma=\tau_{y},
$$

which corresponds to the combination of time-reversal and particle-hole symmetries [see Appendix B for more details]. As these symmetries do not depend on any particular crystal structure, the chiral symmetry specifies the most general symmetry-protected topological number [14]. In the present case, the general topological number is the $3 \mathrm{D}$ winding number

$w_{3 \mathrm{D}}=\int \frac{d^{3} k}{48 \pi^{2}} \epsilon^{\alpha \beta \gamma} \operatorname{tr}\left[\Gamma H^{-1} \partial_{\alpha} H H^{-1} \partial_{\beta} H H^{-1} \partial_{\gamma} H\right]$.

Here, we calculate $w_{3 \mathrm{D}}$ for the $A_{1 u}$ state with $\Delta=\Delta_{0} \sigma_{x}$. In particular, we consider the weak pairing limit $\Delta_{0} / \mu \ll 1$, similar to ordinary superconductors.

First, we numerically evaluate $w_{3 \mathrm{D}}$. It is observed that, when the absolute value of the chemical potential $|\mu|$ is less than the critical value $\mu_{c}$,

$$
\mu_{c}=\sqrt{m_{0}^{2}+\Delta_{0}^{2}}
$$

$w_{3 \mathrm{D}}$ is zero; i.e.,

$$
w_{3 \mathrm{D}}\left(|\mu|<\mu_{c}\right)=0 \text {. }
$$

At $|\mu|=\mu_{c}$, the BdG Hamiltonian in Eq. (13) becomes gapless at $k=0$; thus, the topological phase transition takes place at $\mu=\mu_{c}$. Then, for $|\mu|>\mu_{c}$, we have

$w_{3 \mathrm{D}}\left(|\mu|>\mu_{c}\right)= \begin{cases}-2 \operatorname{sgn}\left(v_{1}\right) & \text { for } \frac{v_{2}}{v_{1}}<-\frac{1}{3}, \\ 4 \operatorname{sgn}\left(v_{1}\right) & \text { for }-\frac{1}{3}<\frac{v_{2}}{v_{1}}<\frac{1}{2}, \\ -4 \operatorname{sgn}\left(v_{1}\right) & \text { for } \frac{1}{2}<\frac{v_{2}}{v_{1}}<3, \\ 2 \operatorname{sgn}\left(v_{1}\right) & \text { for } \frac{v_{2}}{v_{1}}>3 .\end{cases}$

For $|\mu|>\mu_{c}$, the system supports two Fermi surfaces (inner and outer Fermi surfaces) around the $\Gamma$ point, as illustrated in Fig. 3. When $w_{3 \mathrm{D}}$ changes at $v_{2} / v_{1}=-1 / 3,3$ $\left(v_{2} / v_{1}=1 / 2\right)$, six (eight) point nodes appear on the outer Fermi surface, each of which contributes to the change of $w_{3 \mathrm{D}}$ by $1(-1)$ with the increase in $v_{2}$. These results indicate that the $A_{1 u}$ state hosts nontrivial topological superconductivity when $|\mu|>\mu_{c}$. In particular, it supports a characteristic higher winding number $\left|w_{3 \mathrm{D}}\right| \geq 1$. As discussed immediately below, the higher winding number of the topological superconductivity is a direct consequence of the higher spin of the present system.

Here, we consider the origin of the higher winding number. Usually, the pairing interaction is too weak to mix the energetically separated bands. In this weak-pairing case, the topological number of the superconductor is attributed to that of each band forming Fermi surfaces, 
where the electron and hole states are degenerated. The contribution from each band remains unchanged unless the gap of the system closes or the Fermi surfaces contact each other [61]. In the present case, we have [62]

$$
w_{3 \mathrm{D}}=\frac{1}{2} \sum_{n} \operatorname{sgn}\left[v_{F}^{n}\right] \operatorname{sgn}\left[d_{n}\right] \nu_{\mathrm{Ch}}^{n},
$$

where $n$ is summed for all band indices forming disconnected Fermi surfaces, $v_{F}^{n}$ is the Fermi velocity on the $n$th Fermi surface, and $d_{n}$ is the expectation value of the gap function

$$
d_{n}=\left\langle u_{n, k}|\Delta| u_{n, k}\right\rangle
$$

the ket vector $\left|u_{n, k}\right\rangle$ is the solution of Bloch equation $H_{0}(\boldsymbol{k})\left|u_{n, \boldsymbol{k}}\right\rangle=0$ on the $n$th Fermi surface, and $\nu_{\mathrm{Ch}}$ is the first Chern number of the Fermi surface,

$$
\nu_{\mathrm{Ch}}^{n}=-i \int_{k=k_{F}^{n}} d^{2} k^{\prime} \epsilon^{\alpha \beta}\left\langle\partial_{k_{\alpha}^{\prime}} u_{n, k} \mid \partial_{k_{\beta}^{\prime}} u_{n, k}\right\rangle,
$$

where $k_{\alpha}^{\prime}$ represents the two-dimensional momenta on the Fermi surface. Here, we consider Kramers partners separately in the summation of $n$. See Appendix B for more details.

The relation between the $J=3 / 2$ spin and the higher winding number becomes evident in the spherical symmetric case at $v_{2}=0$. In this case, the solution of the Bloch equation $H_{0}(\boldsymbol{k})\left|u_{j_{z}, \pm, k}\right\rangle=\xi_{j_{z}, \pm}\left|u_{j_{z}, \pm, k}\right\rangle$ for the normal state is given by

$$
\left|u_{j_{z}, \pm, k}\right\rangle=R(\hat{\boldsymbol{k}}, \boldsymbol{J})\left|j_{z}\right\rangle \otimes\left|\Psi_{ \pm}\left(\rho_{k}\right)\right\rangle_{\sigma} .
$$

Here, $R(\hat{\boldsymbol{k}}, \boldsymbol{j})=e^{-i J_{z} \phi_{k}} e^{-i J_{y} \theta_{k}}$ with the polar and azimuthal angles $\left(\theta_{k}, \phi_{k}\right)$ of $\boldsymbol{k}$ is the rotation matrix that diagonalizes the spin-dependent part of $H_{0}(\boldsymbol{k})$ as $R^{\dagger}(\hat{\boldsymbol{k}}, \boldsymbol{J}) \boldsymbol{k} \cdot \boldsymbol{J} R(\hat{\boldsymbol{k}}, \boldsymbol{J})=$ $k J_{z}$ and $\left|j_{z}\right\rangle \in\{| \pm 3 / 2\rangle,| \pm 1 / 2\rangle\}$ is the eigenstate of $J_{z}$. The orbital-dependent part of the wave functions is given by

$$
\left|\Psi_{ \pm}\left(\rho_{k}\right)\right\rangle_{\sigma}=e^{-i \sigma_{y} \rho_{k}}| \pm\rangle
$$

with $\rho_{k}=\frac{1}{2} \arctan \left[v_{1} k j_{z} / m(k)\right]$ and the eigenstate $| \pm\rangle$ of $\sigma_{z}$. It is observed that the states $\left|u_{j_{z},+, k}\right\rangle\left(\left|u_{j_{z},-, k}\right\rangle\right)$ yield the electron (hole) branches of the normal spectra, and, hence, they define the Fermi surfaces for $\mu>\mu_{c}\left(\mu<-\mu_{c}\right)$. Therefore, Eq. (24) leads to

$$
w_{3 \mathrm{D}}=\sum_{j_{z}} w_{3 \mathrm{D}}^{j_{z}}
$$

with

$$
w_{3 \mathrm{D}}^{j_{z}}= \pm \frac{1}{2} \operatorname{sgn}\left(d_{j_{z}, \pm}\right) \nu_{\mathrm{Ch}}^{j_{z}}
$$

where the double sign \pm corresponds to the case with $\mu \gtrless \pm \mu_{c}, d_{j_{z}, \pm}=\left\langle u_{j_{z}, \pm, k}\left|\Delta_{0} \sigma_{x}\right| u_{j_{z}, \pm, k}\right\rangle$, and $\nu_{\mathrm{Ch}}^{j_{z}}$ is the first Chern number of $\left|u_{j_{z}, k}^{ \pm}\right\rangle$on each Fermi surface. Equation (27) yields

$$
\operatorname{sgn}\left(d_{j_{z}, \pm}\right)= \pm \operatorname{sgn}\left(j_{z} v_{1}\right)
$$

Using the polar coordinates of the Fermi surface, we can also evaluate $\nu_{\mathrm{Ch}}^{j_{z}}$ analytically:

$$
\begin{aligned}
\nu_{\mathrm{Ch}}^{j_{z}} & =\frac{1}{2 \pi} \int d \theta_{k} d \phi_{k}\left(-i\left\langle\frac{\partial u_{j_{z}, k}^{ \pm}}{\partial \theta_{k}} \mid \frac{\partial u_{j_{z}, k}^{ \pm}}{\partial \phi_{k}}\right\rangle+\text { H.c. }\right) \\
& =2 j_{z} .
\end{aligned}
$$

Thus, we have

$$
w_{3 \mathrm{D}}^{j_{z}}=\operatorname{sgn}\left(v_{1}\right)\left|j_{z}\right|,
$$

for both $\mu>\mu_{c}$ and $\mu<\mu_{c}$.

The last expression indicates that a higher spin provides a higher winding number. Using this expression, we can also evaluate the winding number for each Fermi surface. For $v_{2}=0$, the outer (inner) Fermi surface consists of $j_{z}=$ $\pm 1 / 2\left(j_{z}= \pm 3 / 2\right)$ components, and, hence, we obtain

$$
\begin{gathered}
w_{\text {out }}=w_{3 \mathrm{D}}^{1 / 2}+w_{3 \mathrm{D}}^{-1 / 2}=\operatorname{sgn}\left(v_{1}\right), \\
w_{\mathrm{in}}=w_{3 \mathrm{D}}^{3 / 2}+w_{3 \mathrm{D}}^{-3 / 2}=3 \operatorname{sgn}\left(v_{1}\right),
\end{gathered}
$$

where $w_{\text {out }}\left(w_{\text {in }}\right)$ denotes the winding number of the outer (inner) Fermi surface. These winding numbers retain the same values for $-1 / 3<v_{2} / v_{1}<1 / 2$, as either a gap closing or a contact of Fermi surfaces does not occur until $v_{2} / v_{1}$ reaches the boundary of the region at $v_{2} / v_{1}=1 / 2$ or $-1 / 3$. Here, we note that the total winding number $w_{\text {out }}+w_{\text {in }}$ reproduces the numerical result of Eq. (23) in the same region of $-1 / 3<v_{2} / v_{1}<1 / 2$.

Using the above result, we can also identify the winding numbers $\left(w_{\text {out }}, w_{\text {in }}\right)$ for the other regions of $v_{2} / v_{1}$. First, to evaluate them in the region $1 / 2<v_{2} / v_{1}<3$, we use a "duality" relation. As explained in Appendix C, a unitary transformation maps the parameter $\left(v_{1}, v_{2}\right)$ to $\left(-3 v_{1} / 5-4 v_{2} / 5,-4 v_{1} / 5+3 v_{2} / 5\right)$. This transformation exchanges the region of $-1 / 3<v_{2} / v_{1}<1 / 2$ with that of $1 / 2<v_{2} / v_{1}<3$, retaining the energy spectra and reversing the winding numbers. Thus, combining the duality relation with Eq. (34), we obtain

$$
w_{\text {out }}=-\operatorname{sgn}\left(v_{1}\right), \quad w_{\text {in }}=-3 \operatorname{sgn}\left(v_{1}\right),
$$

for $1 / 2<v_{2} / v_{1}<3$. Subsequently, to determine the winding numbers for the remaining two regions $v_{2} / v_{1}<-1 / 3$ and $v_{2} / v_{1}>3$, we use the properties of the topological 
phase transitions at $v_{2} / v_{1}=-1 / 3$ and $v_{2} / v_{1}=3$. As mentioned above, at the topological phase transitions, six point nodes appear on the outer Fermi surface, which change $\left|w_{\text {out }}\right|$ by 6 [63]. Provided also that the total winding number $w_{\text {out }}+w_{\text {in }}$ reproduces Eq. (23), we can uniquely determine the winding numbers as

$$
w_{\text {out }}=-5 \operatorname{sgn}\left(v_{1}\right), \quad w_{\text {in }}=3 \operatorname{sgn}\left(v_{1}\right),
$$

for $v_{2} / v_{1}<-1 / 3$, and

$$
w_{\text {out }}=5 \operatorname{sgn}\left(v_{1}\right), \quad w_{\text {in }}=-3 \operatorname{sgn}\left(v_{1}\right),
$$

for $v_{2} / v_{1}>3$. In both cases, the Fermi surfaces have characteristic higher winding numbers.

\section{B. Mirror Chern numbers}

The present system has the $O_{h}$-group crystalline symmetry, which provides more detailed topological information [64-68]. Specifically, we first consider the horizontal (or, equivalently, vertical) mirror reflection $M_{x}$ with respect to the $k_{x}$ axis.

The $A_{1 u}$ gap function is odd under the mirror reflection. In this case, the mirror reflection operator for the $\mathrm{BdG}$ Hamiltonian is given by $M \tau_{z}$, where $M=P C_{2, n}$ is the mirror operator for the normal Hamiltonian $H_{0}(\boldsymbol{k})$ and $\boldsymbol{n}$ is the normal vector of the reflection plane [69]. On the mirror-invariant plane $\boldsymbol{k} \cdot \boldsymbol{n}=0$, the BdG Hamiltonian can be block diagonal in the diagonal basis of $M \tau_{z}$. Thus, the mirror Chern number $\tilde{\nu}_{\sigma_{h, d}}$ for the BdG Hamiltonian is defined in the same manner as $\nu_{\sigma_{h, d}}$ in Sec. II.

First, let us evaluate $\tilde{\nu}_{\sigma_{h}}$ in the limit $\mu=0$ and $\Delta_{0}=0$ for the mirror reflection with respect to three equivalent horizontal planes: $x=0, y=0$, or $z=0$. In this limit, the BdG Hamiltonian reduces to $H_{0}(\boldsymbol{k}) \tau_{z}$, and, hence, $\tilde{\nu}_{\sigma_{h}}$ can be evaluated as $\tilde{\nu}_{\sigma_{h}}=2 \nu_{\sigma_{h}}$. Therefore, from Eq. (10), we have

$$
\tilde{\nu}_{\sigma_{h}}(\mu=0)=4 \operatorname{sgn}\left(b_{3 / 2} b_{1 / 2}\right) .
$$

For small $\Delta_{0}$ and $\mu, \tilde{\nu}_{\sigma_{h}}$ retains the same value unless the gap of the system closes.

When $|\mu|=\mu_{c}$, the gap of the system closes at the $\Gamma$ point, and $\tilde{\nu}_{\sigma_{h}}$ changes. Using the fourfold rotation symmetry around the $k_{x}$ axis, we obtain the following useful formula to calculate $\tilde{\nu}_{\sigma_{h}}[35]$ :

$$
\tilde{\nu}_{\sigma_{h}}=\sum_{j_{x}} N_{j_{x},+}(\Gamma) j_{x} \bmod 4,
$$

where $N_{j_{x},+}(\Gamma)$ is the number of negative-energy states with the spin $j_{x}$ at the $\Gamma$ point in the eigensector of $M_{x} \tau_{z}$ with the eigenvalue $\lambda_{M_{x} \tau_{z}}=+i$. In the weak-coupling limit $\Delta_{0} \rightarrow 0$, the particle and hole sectors are decoupled so that
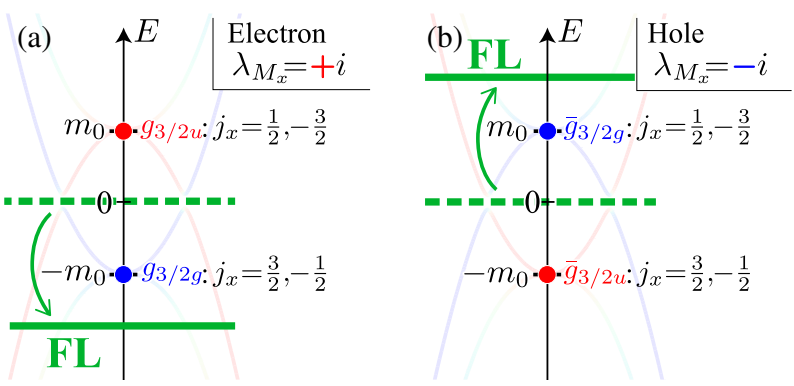

FIG. 6. Level structures and their angular momentum in the $\lambda_{M_{x} \tau_{z}}=i$ sector at the $\Gamma$ point. (a) and (b) indicate electron and hole sectors, respectively.

$\lambda_{M_{x} \tau_{z}}=\lambda_{M_{x}} \lambda_{\tau_{z}}$. In this case, a negative-energy state in the $\lambda_{M_{x} \tau_{z}}=i$ sector reduces to either an electron state $\left(\lambda_{\tau_{z}}=+1\right)$ in the $\lambda_{M_{x}}=i$ sector or a hole state $\left(\lambda_{\tau_{z}}=-1\right)$ in the $\lambda_{M_{x}}=-i$ sector below the Fermi level. Therefore, $N_{j_{x},+}(\Gamma)$ can be evaluated as the number of these electron and hole states. Thus, as summarized in Fig. 6, when $|\mu|$ exceeds $m_{0}$, the $j_{x}=3 / 2,-1 / 2$ electron $\left(j_{x}=1 / 2,-3 / 2\right.$ hole $)$ bands in the $\lambda_{M_{x}}=i\left(\lambda_{M_{x}}=-i\right)$ sector move above (below) the Fermi level at $\Gamma$. Therefore, the mirror Chern number jumps by

$$
\begin{aligned}
\Delta \tilde{\nu}_{\sigma_{h}} & =-\left(\frac{3}{2}-\frac{1}{2}\right)+\left(\frac{1}{2}-\frac{3}{2}\right) \bmod 4 \\
& =-2 \bmod 4
\end{aligned}
$$

We can also evaluate $\tilde{\nu}_{\sigma_{h}}$ numerically as

$$
\tilde{\nu}_{\sigma_{h}}\left(|\mu|>\mu_{c}\right)=2 \operatorname{sgn}\left(b_{3 / 2} b_{1 / 2}\right),
$$

which is consistent with Eq. (40).

According to the bulk-edge correspondence, the nonzero mirror Chern number indicates the existence of surface states. Here, we calculate the energy spectrum in the slab geometry in Fig. 7 with a finite size $L$ along the $z$ axis. This system has horizontal (diagonal) mirror reflection symmetry with respect to the $x(x+y)$ direction even in the presence of the surface. Along the high symmetric line

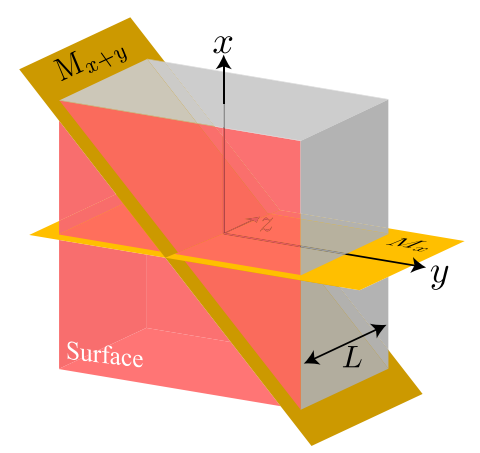

FIG. 7. System geometry of the slab system. 
$k_{x}=0 \quad\left(k_{x}+k_{y}=0\right)$, we numerically solve the $\mathrm{BdG}$ equation for each mirror sector by replacing the momentum $k_{z} \rightarrow-i \partial_{z}$ and using the Gauss-Lobatto expansion method [70].

As shown in the left column in Fig. 8, when $|\mu|<\mu_{c}$, the spectrum along the $k_{x}=0$ line exhibits four branches of surface states. The number of branches coincides with $\left|\tilde{\nu}_{\sigma_{h}}\right|$. Furthermore, the surface states change the chirality (or the connectivity with the bulk states) when $\tilde{\nu}_{\sigma_{h}}$ in the bulk changes the sign. For $-1 / 3<v_{1} / v_{2}<3$ [see Figs. 8(b)
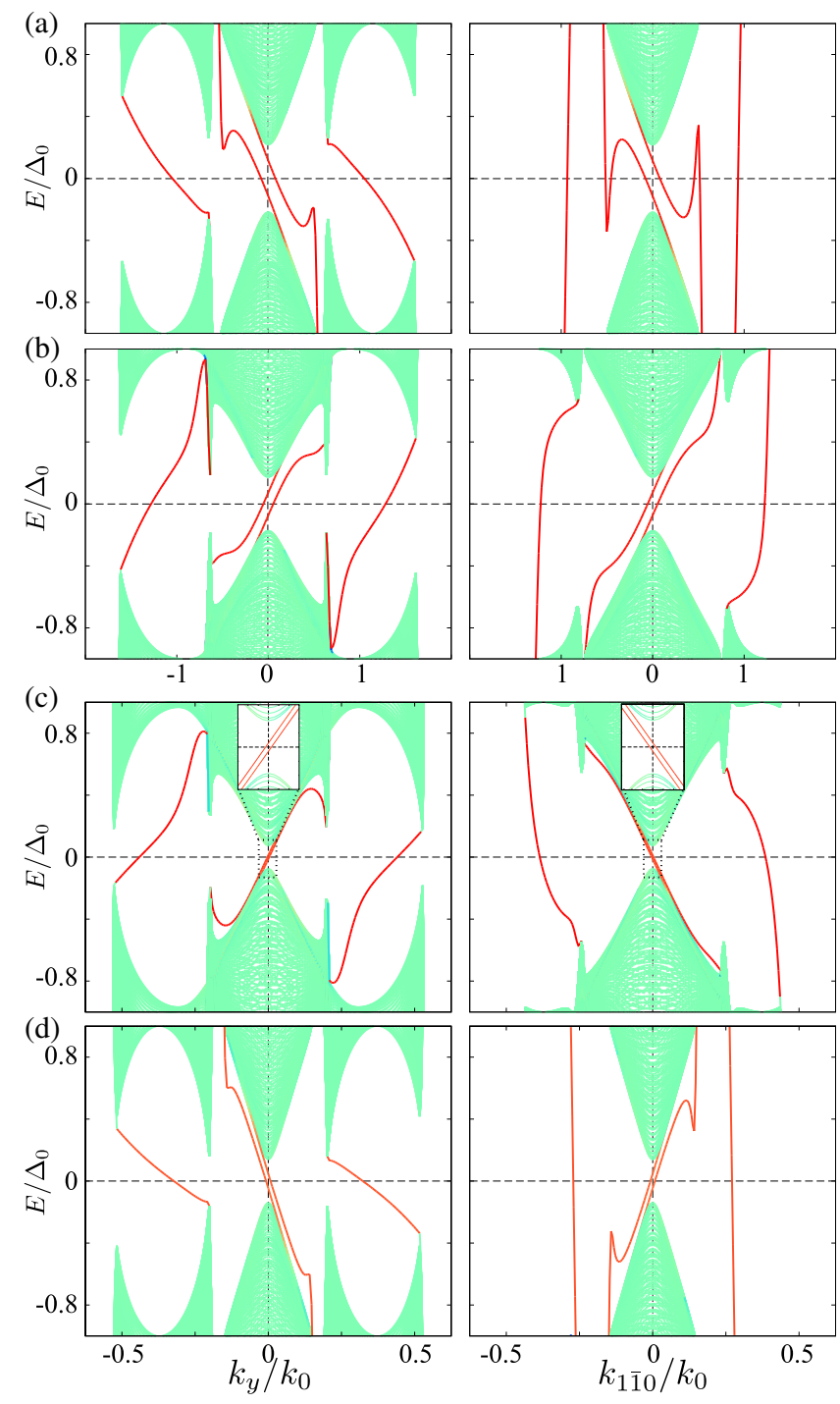

FIG. 8. Energy spectrum of the slab in different topological phases of a small $|\mu|$ regime along the $k_{x}$ (left) and $k_{1 \overline{1} 0}=$ $\left(k_{x}-k_{y}\right) / \sqrt{2}$ (right) directions. For better illustration, we display the surface states localized only at $z=-L / 2$ highlighted by the red curves. The momentum unit is $k_{0}=m_{0} / v_{1}$. Insets in (b) are magnified images of the center of the Brillouin zone and close to $E=0$. Here, $\Delta_{0}=0.025 m_{0}, \mu=-0.75 m_{0}, v_{2} / v_{1}=-0.5$ (a), -0.2 (b), 2.5 (c), and 4 (d), and $\alpha=0.64 v_{1}^{2} / m_{0}$ (a),(b) and $6.25 v_{1}^{2} / m_{0}(\mathrm{c}),(\mathrm{d})$. and 8(c)], each surface state connects the bulk bands upward with the increase in $k_{y}$, but for $v_{1} / v_{2}<-1 / 3$ or for $v_{1} / v_{2}>3$ [see Figs. 8(a) or 8(d)], it connects them downward. A similar bulk-edge correspondence holds for the higher-doped phase $|\mu|>\mu_{c}$ as shown in Fig. 9. In this case, when the surface state cuts the zero energy upward (downward) with the increase in $k_{y}$, it accumulates the mirror Chern number by $1(-1)$. The total mirror Chern number counted from Fig. 9 is consistent with Eq. (41).

Similarly, the diagonal mirror Chern number $\tilde{\nu}_{\sigma_{d}}$ with respect to the $k_{x}+k_{y}$ direction can be defined for the $\mathrm{BdG}$


FIG. 9. Energy spectrum of the slab in different topological phases for a large $|\mu|$ regime, $\mu=-1.25 m_{0}$. The red and blue symbols denote the zero-energy state of helical Majorana fermions originating from the outer and inner Fermi surfaces, respectively. The closed and open ones indicate the positive and negative helicities, respectively. The open square symbols indicate pairs of helical Majorana fermions with opposite helicities, which can annihilate via continuous deformation. The other conditions are the same as in Fig. 8. 
Hamiltonian. For $\mu=0$, it is evaluated as twice the value of that in Fig. 1(f), which persists as long as $\mu$ reaches the critical value $\mu_{c}$ :

$$
\tilde{\nu}_{\sigma_{d}}\left(|\mu|<\mu_{c}\right)= \begin{cases}+4 & \text { for }-\frac{1}{3}<\frac{v_{2}}{v_{1}}<\frac{1}{2} \\ -4 & \text { for } \frac{1}{2}<\frac{v_{2}}{v_{1}}<3 \\ 0 & \text { otherwise. }\end{cases}
$$

In addition, for $|\mu|>\mu_{c}$, we numerically determine that

$$
\tilde{\nu}_{\sigma_{d}}\left(|\mu|>\mu_{c}\right)= \begin{cases}+2 & \text { for }-\frac{1}{3}<\frac{v_{2}}{v_{1}}<\frac{1}{2} \\ -2 & \text { for } \frac{1}{2}<\frac{v_{2}}{v_{1}}<3 \\ 0 & \text { otherwise }\end{cases}
$$

The quasiparticle spectra along the mirror-invariant line $k_{x}+k_{y}=0$ for the slab geometry are shown in the right columns in Figs. 8 and 9, all of which are consistent with the above values of the diagonal mirror Chern number. In particular, for $\mu=-0.75 m_{0}$, the surface dispersions of Figs. 8(a) and 8(d) cut the zero energy upward as many times as downward, from right to left, and, hence, there is no topological protection.

In Fig 9, all the surface states for $|\mu|>\mu_{c}$ pass through the high-symmetry point $k_{y}=0\left(k_{1} \overline{0}=0\right)$ at $E=0$. The origin of this property is explained in the next section.

\section{1D winding numbers}

The system also has fourfold rotation symmetry, from which we can define another topological invariant. The $A_{1 u}$ gap function is invariant under the fourfold rotation along the primary axis, and, hence, the BdG Hamiltonian trivially realizes the fourfold symmetry as

$\tilde{C}_{4, z} H\left(k_{y},-k_{x}, k_{z}\right) \tilde{C}_{4, z}^{-1}=H(\boldsymbol{k}), \quad \tilde{C}_{4, z}=C_{4, z} \tau_{0}$.

On the primary axis $k_{x}=k_{y}=0$, the BdG Hamiltonian is block diagonal in the eigenbasis of $\tilde{C}_{4, z}$ with the eigenvalue $\lambda_{\tilde{C}_{4, z}}=e^{-i\left(j_{z} / 2\right) \pi}$ :

$$
H_{j_{z}}\left(k_{z}\right)=H_{0, j_{z}}\left(k_{z}\right) \tau_{z}+\Delta_{0} \sigma_{x} \tau_{x},
$$

where $H_{0, j_{z}}\left(k_{z}\right)$ is given in Eq. (5). We also observe that each sector $H_{j_{z}}\left(k_{z}\right)$ retains chiral symmetry $\Gamma=i \mathcal{T C}$ in Eq. (B8), as it holds that $\left[\Gamma, \tilde{C}_{4, z}\right]=0$. Thus, on the basis where $\Gamma$ is diagonal, $H_{j_{z}}\left(k_{z}\right)$ takes the form

$$
H_{j_{z}}=\left(\begin{array}{cc}
0 & h_{j_{z}}\left(k_{z}\right) \\
h_{j_{z}}^{\dagger}\left(k_{z}\right) & 0
\end{array}\right) .
$$

Thus, we can introduce the $1 \mathrm{D}$ winding number

$$
w_{1 \mathrm{D}}^{j_{z}}=\frac{1}{2 \pi} \operatorname{Im}\left\{\int d k_{z} \partial_{k_{z}} \ln \left[\operatorname{det}\left(h_{j_{z}}\right)\right]\right\},
$$

with

$\operatorname{det}\left(h_{j_{z}}\right)=\mu^{2}-m_{j_{z}}^{2}\left(k_{z}\right)+\left(b_{j_{z}} k_{z}\right)^{2}-\Delta_{0}^{2}+2 i b_{j_{z}} k_{z} \Delta_{0}$,

where $b_{j_{z}}$ is given by Eqs. (6) and (7). It can be calculated analytically as

$$
w_{1 \mathrm{D}}^{j_{z}}= \begin{cases}0 & \text { for }|\mu|<\mu_{c}, \\ \operatorname{sgn}\left(b_{j_{z}}\right) & \text { for }|\mu|>\mu_{c} .\end{cases}
$$

It is also observed that the total winding number vanishes, $\sum_{j_{z}} w_{1 \mathrm{D}}^{j_{z}}=0$, because of $b_{-j_{z}}=-b_{j_{z}}$, and the $1 \mathrm{D}$ winding number is related to the mirror Chern number as

$$
2 w_{1 \mathrm{D}}^{3 / 2} w_{1 \mathrm{D}}^{1 / 2}=\tilde{\nu}_{\sigma_{h}} \bmod 4 .
$$

When $|\mu|>\mu_{c},\left|w_{1 \mathrm{D}}^{j_{z}}\right|=1$ for each $j_{z}$, which indicates that there must be four zero modes at $k_{x}=k_{y}=0$ in each mirror subsector, which explains why the surface states in one mirror sector in Fig. 9 pass the high symmetric points $k_{y}=0$ or $k_{1 \overline{1} 0}=0$ when $\mu=-1.25 m_{0}$.

\section{Topological phase diagram}

We summarize the obtained topological phase diagram in Fig. 10. On the bottom of Fig. 10 with $|\mu|=0$, the topological indices and the corresponding surface states originate from those in the normal state. As already discussed in Sec. II, octahedral or cubic Dirac points appear at topological phase transition points in this regime. In contrast, for $|\mu|>\mu_{c}$, as shown in Fig. 10(f) [Figs. 10(e) and $10(\mathrm{~g})$ ], eight (six) point nodes of the superconducting gap appear on the outer Fermi surface at $v_{2}=v_{1} / 2\left(3 v_{1}\right.$ and $\left.-v_{1} / 3\right)$. These point nodes are also caused by the topological phase transitions where the bulk topological number $w_{3 \mathrm{D}}$ changes by eight (six). These kinds of nodal structures are overlooked in the group theoretical classification of gap nodes [71], but our topological analysis clarifies that the presence of the point nodes is not accidental at the same level as the bulk Dirac points in the normal states. The presence of point dips near the topological phase transitions provides a chance to confirm the $A_{1 u}$ state through low-temperature measurements of the specific heat, nuclear magnetic resonance spectrum, etc.

In Fig. 10, we also illustrate patterns of helical Majorana fermions composed of electrons of the outer and inner Fermi surfaces. The plus and minus signs in Fig. 10 denote the helicities of the Majorana fermions, where the total helicities for the outer (inner) Fermi surface should be the same as the winding number $w_{\text {out }}\left(w_{\text {in }}\right)$. As explained 


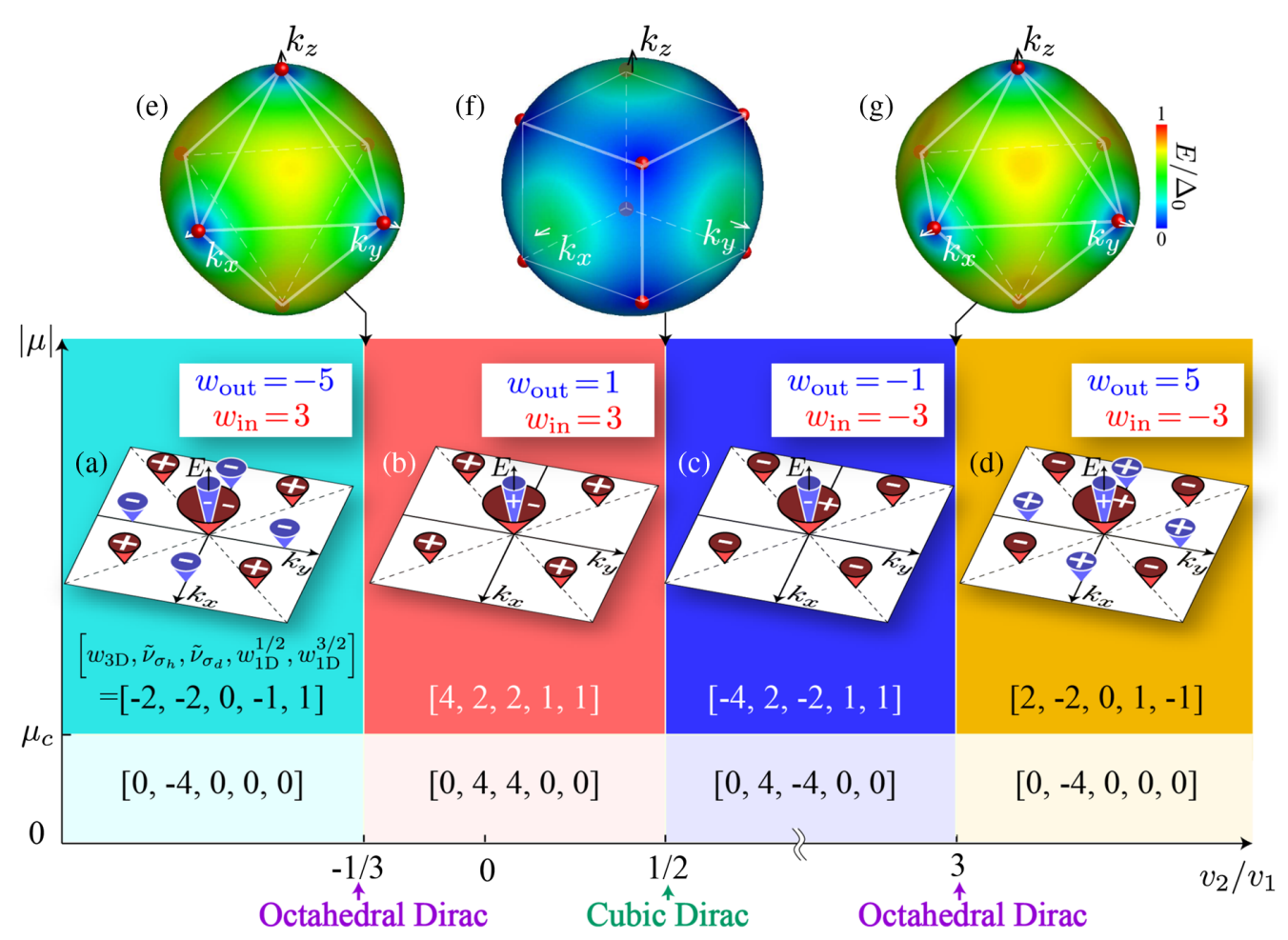

FIG. 10. Topological phase diagram of the $A_{1 u}$ pairing state for $v_{1}>0$. The $A_{1 u}$ state may host the $3 \mathrm{D}$ winding number $w_{3 \mathrm{D}}$ as a timereversal-invariant superconductor. From the $O_{h}$-group symmetry, it also supports the two different mirror Chern numbers $\tilde{\nu}_{\sigma_{h}}$ and $\tilde{\nu}_{\sigma_{d}}$ and the rotation $1 \mathrm{D}$ winding number $w_{1 \mathrm{D}}^{j_{z}}$. If we consider $v_{1}<0$, the winding numbers $w_{3 \mathrm{D}}$ and $w_{1 \mathrm{D}}^{j_{z}}$ change their signs. The insets indicate the location of a helical Majorana fermion in the surface Brillouin zone, which carries by the winding number \pm 1 . The cones with blue and red in the $|\mu|>\mu_{c}$ phase show the contributions from the outer and inner Fermi surfaces, respectively. These colors correspond to the symbols in Fig. 9. The gap structures on the outer Fermi surface (e) $v_{2}=3 v_{1}$, (f) $v_{2}=v_{1} / 2$, and (g) $v_{2}=v_{1} / 2$. The red dots indicate the point node of the superconducting gap. Here, $\mu=-2 m_{0}, \beta_{i}=0, m_{0}>0$, and $\alpha=6.25 v_{1}^{2} / m_{0}$ for (e),(f) and $\alpha=$ $0.64 v_{1}^{2} / m_{0}$ for $(\mathrm{g})$.

below, these configurations are determined by comparing the surface state spectra in Fig. 9 with the winding numbers and considering the requirement from symmetry.

Let us first consider the region $-1 / 3<v_{2} / v_{1}<1 / 2$, which includes the spherical symmetric point at $v_{2}=0$. See Figs. 9(b) and 10(b). In this region, the outer Fermi surface with $w_{\text {out }}=1$ provides a single helical Majorana fermion at the center of the surface Brillouin zone, which we denote as the blue symbols and cones in Figs. 9(b) and 10(b), respectively, at $k=0\left(k=k_{y}\right.$ or $\left.k_{1 \overline{1} 0}\right)$. The inner Fermi surface has an unusual higher winding number $w_{\text {in }}=3$. In the spherical symmetric limit $v_{2}=0$, we have a single surface state at the center of the surface Brillouin zone, but the surface spectrum shows a higher-order $k$ dependence near $k=0$. Moreover, when $v_{2} \neq 0$, the surface state breaks into several helical Majorana fermions, because the spherical symmetry reduces to the point group $O_{h}$. For consistency with $C_{4 v}$ symmetry on the surface, there should be at least a single helical Majorana fermion at the center of the surface Brillouin zone and four others at other points, as illustrated in Fig. 10(b). In terms of the winding number, this splitting corresponds to $w_{\text {in }}=-1+4$. From the actual surface spectrum in Fig. 9(b), it is observed that the four
Majorana fermions are located on the diagonal mirror lines in the surface Brillouin zone. We also observe that the two center helical Majorana fermions on the inner and outer Fermi surfaces do not mix, although they are located at the same position with opposite helicities, which is because they also have different topological numbers: $w_{1 \mathrm{D}}^{1 / 2}=w_{1 \mathrm{D}}^{3 / 2}=1$.

To generate the center and satellite Majorana fermions simultaneously, the surface state has nonmonotonic dispersion. We can observe this behavior in Fig. 9(b), for example, where a surface state connecting the bulk bands from $k_{1 \overline{1} 0} \sim-0.7 k_{0}$ to $0.7 k_{0}$ cuts the zero energy upward in $k_{1 \overline{1} 0}<0$, downward at $k_{1 \overline{1} 0}=0$, and upward again in $k_{1 \overline{1} 0}>0$. This nonmonotonic behavior enhances the density of states around $E=0$, providing a characteristic zero-bias conductance peak in the tunnel conductance spectra [72].

We can also determine the configurations of surface Majorana fermions in other regions. Similar to the argument for the bulk winding numbers in Sec. IVA, we use the duality relation to determine the configuration in the region $1 / 2<v_{2} / v_{1}<3$. As the duality retains the spectrum but reverses the winding numbers, the configuration in Fig. 10(c) is obtained from Fig. 10(b). Finally, the configurations in Figs. 10(a) and 10(d) are determined by considering the 
topological phase transitions at $v_{2} / v_{1}=-1 / 3$ and 3. At these transition points, six point nodes appear on the outer Fermi surface. Upon being projected on the surface Brillouin zone, these point nodes change the helicity of the center Majorana fermions by two and create four additional satellite Majorana fermions in the outer Fermi surface excitations, as shown in Figs. 10(a) and 10(d). The obtained Majorana configurations in Figs. 10(a), 10(c), and 10(d) are consistent with the spectra in Figs. 9(a), 9(c), and $9(\mathrm{~d})$, respectively.

\section{DISCUSSION}

\section{A. Application to $\mathrm{Sr}_{3-x} \mathrm{SnO}$}

Notably, a class of antiperovskite materials $A_{3} B X$ with $A=(\mathrm{Ca}, \mathrm{Sr}, \mathrm{La}), B=(\mathrm{Pb}, \mathrm{Sn})$, and $X=(\mathrm{C}, \mathrm{N}, \mathrm{O})$ realizes a higher-spin system. Near the Fermi level, they host a $d$-orbital band of the $A$ atom and a $p$-orbital band of the $B$, which belong to the $G_{3 / 2 g}$ and $G_{3 / 2 u}$ representations of the $O_{h}$ group, respectively. Therefore, our theory is directly applicable to antiperovskite materials [73].

The first-principles calculations for $\mathrm{Sr}_{3} \mathrm{SnO}$ are carried out based on different packages: VASP [52], WIEN2k, and AkaiKKR [56]. These results have scheme dependence in the detailed band structure, such as behavior near the Brillouin zone boundary. However, at least, the presence of band inversion at the $\Gamma$ point is consistent in all the schemes.

The first-principles calculations show that the antiperovskites support the octahedral Dirac points with a tiny gap in the $j_{z}=3 / 2$ sector [50-52]. Therefore, they are near the topological phase transition at $v_{2}=3 v_{1}$ [52]. In addition, in the antiperovskite with valency $\left(A^{+2}\right)_{3}\left(B^{-4}\right)\left(X^{-2}\right)$, $p$ orbitals of $B^{-4}$ ions are almost filled, but $d$ orbitals of $A^{+2}$ are almost empty [50-52,54]. Therefore, in the antiperovskites with a stoichiometric ratio, the Dirac point is pinned near the Fermi level, corresponding to $\mu=0$ in our model Hamiltonian in Eq. (4).

Recently, the superconductivity of antiperovskites has been experimentally observed in $\mathrm{Sr}_{3-x} \mathrm{SnO}$ with the deficiency $x[54,55,57]$. This deficiency yields hole doping. The Fermi level is expected to be much lower than the fourfold degenerate point at $\Gamma$. Therefore, this system can realize the high winding superconductivity in $|\mu|>\mu_{c}$ discussed in the present work if the interorbital attractive interaction is dominant.

Finally, we discuss possible experimental signals of high winding topological superconductivity in the case of $\mathrm{Sr}_{3-x} \mathrm{SnO}$. As mentioned before, $\mathrm{Sr}_{3-x} \mathrm{SnO}$ corresponds to our model with $v_{2} / v_{1} \sim 3$ and $\mu<-\mu_{c}$. This correspondence indicates that, if the system realizes the $J=0$ odd-parity superconductivity, $\mathrm{Sr}_{3-x} \mathrm{SnO}$ is in the vicinity of the topological phase transition between the $w_{\text {out }}=-1$ phase and the $w_{\text {out }}=5$ one in our topological phase diagram in Fig. 10. Therefore, as shown in Figs. 4(a)-4(c), there should be point dips on the outer Fermi surface. Note that there is no gap node on the inner Fermi surface, as the topological phase transition retains the value of $w_{\text {in }}$. The point-node structure can be detected via low-temperature experiments such as heat capacity measurement. Notably, the difference in behaviors of the superconducting gap between the outer and inner Fermi surfaces might explain the double superconducting transition reported in $\mathrm{Sr}_{3-x} \mathrm{SnO}$ [54].

\section{B. Systems without band inversion}

In the present work, for discussing the topological property of normal and $A_{1 u}$ superconducting states, we assume $m_{0} \alpha>0$, where the system exhibits a band inversion of $G_{3 / 2 g}$ and the $G_{3 / 2 u}$ representation at the $\Gamma$ point. In contrast, when $m_{0} \alpha<0$, without band inversion, the normal state is topologically trivial with the mirror Chern number $\nu_{h}=\nu_{d}=0$ in any parameter region of $v_{1}$ and $v_{2}$.

For the $A_{1 u}$ superconducting state in this case, the lowdoped phase $|\mu|<\mu_{c}$ is also topologically trivial, as it can be derived in a manner similar to Sec. IV B. However, in the case without band inversion, the topological phase transition by doping, which shrinks the Fermi surface to one point, occurs at $|\mu|=\mu_{c}$. When the system changes from the low-doped phase $|\mu|<\mu_{c}$ to the high-doped one $|\mu|>\mu_{c}$, the mirror Chern number jumps with the opposite sign to the case with $m_{0} \alpha>0$, as the angular momenta of valence and conduction bands at the $\Gamma$ point (see Fig. 6 for $m_{0} \alpha>0$ ) exhibit opposite signs for the cases with and without band inversion. In addition, applying the analysis in Secs. IVA and IV C, we can derive the 3D and 1D winding numbers with the same value as $m_{0} \alpha>0$. Consequently, all the topological indices for the highdoped phase of $m_{0} \alpha<0$ are the same as those in $m_{0} \alpha>0$, as summarized in Fig. 11.

In contrast, when $m_{0} \alpha<0$, without band inversion, the orbital mixing in both the Fermi surfaces is strongly suppressed, which reduces the transition temperature of the interorbital pairing state $A_{1 u}$. Thus, although the topological property of the high-doped phase is unchanged regardless of the sign of $m_{0} \alpha$, the band inversion plays a key role in obtaining the new topological superconductivity discussed in this work.

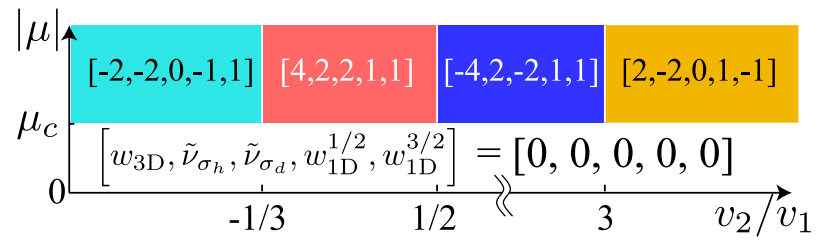

FIG. 11. Topological phase diagram of the $A_{1 u}$ pairing state for $m_{0} \alpha<0$. The other conditions are the same as in Fig. 10. 


\section{Other pairing states}

Depending on the pairing interaction, we cannot avoid the possibility of the $s$-wave superconductivity belonging to the $A_{1 g}$ representation in Table II. As discussed in Sec. III B, it exhibits the highest transition temperature in even-parity superconductivity. In this case, not only the 3D winding number but also the symmetry-protected 1D winding numbers and mirror Chern numbers in the superconducting state are trivial.

However, the $s$-wave superconducting state may host surface topological superconductivity. Owing to the nonzero mirror Chern numbers in the normal state, the system supports surface Dirac fermions in the normal state. In the $s$-wave superconducting state, the surface Dirac fermion exhibits surface topological superconductivity [75].

The $A_{1 u}$ and $A_{1 g}$ states with $J=0$ mainly discussed so far are caused by the Cooper pair between time-reversal partners. However, this is not the only possible partner. An electron may also form a Cooper pair with its inversion partner. Such a Cooper pair inevitably has a parallel spin configuration with $J=1,3$, as inversion does not flip the spin. As shown in Table II, the $A_{2 u}, T_{1 u}$, and $T_{2 u}$ states contain this class of Cooper pairs.

As shown in Fig. 2, $T_{1 u}$ exhibits a relatively higher $T_{c}$. Notably, $T_{c}$ of $T_{1 u}$ is comparable to that of $A_{1 u}$, which suggests that $T_{1 u}$ could win by improving the spin and orbital textures slightly. Indeed, a similar exchange of the pairing symmetry has been reported in the case of doped TIs with spin $1 / 2$. The higher-order term with respect to $k$ enhances the $T_{c}$ of the spin-triplet $E_{u}$ representation of $D_{3 d}$ symmetry more than that of the spin-singlet $A_{1 u}$ one [21]. It supports the experimental observation of the anisotropic gap [22,23]. Similarly, the present calculation does not exclude the possibility of $T_{1 u}$.

Let us consider the gap structure and spin property of the $T_{1 u}$ state. It hosts $J=1$ and $J=3$ components, and, hence, the gap function is given as

$$
\Delta=\frac{2 \Delta_{0}}{\sqrt{5}}\left(\chi_{1} J_{z}+\chi_{3} \tilde{J}_{z}\right) \sigma_{y}
$$

with the normalization condition of the mixing ratio $\chi_{1}^{2}+\chi_{3}^{2}=1$. Projecting Eq. (51) onto the band basis of the normal state, we obtain the energy gap at the Fermi surface of the $n$th band as [see Appendix B]

$$
\begin{aligned}
d_{n}^{\prime}(\boldsymbol{k}) & =-\left\langle u_{n, \boldsymbol{k}}|\Delta| u_{\bar{n}, \boldsymbol{k}}\right\rangle \\
& =\chi_{1} d_{n}^{\prime(1)}(\boldsymbol{k})+\chi_{3} d_{n}^{\prime(3)}(\boldsymbol{k}),
\end{aligned}
$$

where we use the fact that the Cooper pair of the $T_{1 u}$ state is formed by the electron and its inversion partner.

Along the main axis $k_{z}$, the eigenvalue $\lambda_{C_{4}}=e^{-i\left(j_{z} / 2\right) \pi}$ of $C_{4}$ rotation is a good quantum number of the normal state. The bands $n$ and $\bar{n}$ in Eq. (52) belong to a different eigensector with $\lambda_{C_{4}}$ and $-\lambda_{C_{4}}$. In addition, as $J_{z}$ and $\tilde{J}_{z}$ preserve $\lambda_{C_{4}}$, there is a point node $d_{n}^{\prime}\left(k_{z}\right)=0$ at the intersection of the $k_{z}$ axis and the two Fermi surfaces. The presence of the point node is consistent with the grouptheoretical classification of the gap nodes [71]. Along the other momentum direction, adjusting the ratio of these two components $\chi_{1}$ and $\chi_{2}$, the $T_{1 u}$ state can optimize the gap function so as to be consistent with the spin and orbital textures on the Fermi surfaces, as much as possible. We illustrate the optimized superconducting gap of the $T_{1 u}$ state for $v_{2}=3 v_{1}\left(v_{2}=0\right)$ in Figs. 12(c) and 12(d) [Figs. 12(a) and 12(b)].

In Fig. 12(e), we show the parameter dependence of the optimum mixing ratio of the $J=1$ and $J=3$ components at $T_{c}$ in our numerical solution of the gap equation. It is observed that the higher-spin $J=3$ component cannot be neglected in the whole region of $v_{2} / v_{1}$, and it can even dominate the gap function in some regions. Therefore, the $T_{1 u}$ state realizes a higher-spin pairing state. The higherspin nature of the pairing state could be tested via spinsensitive experiments.

In particular, at the rotational symmetric point $v_{2}=0$, the amplitude of the total momentum is a good quantum number. Thus, the $J=1$ state and $J=3$ state do not mix with each other. Let us compare the stability of these two
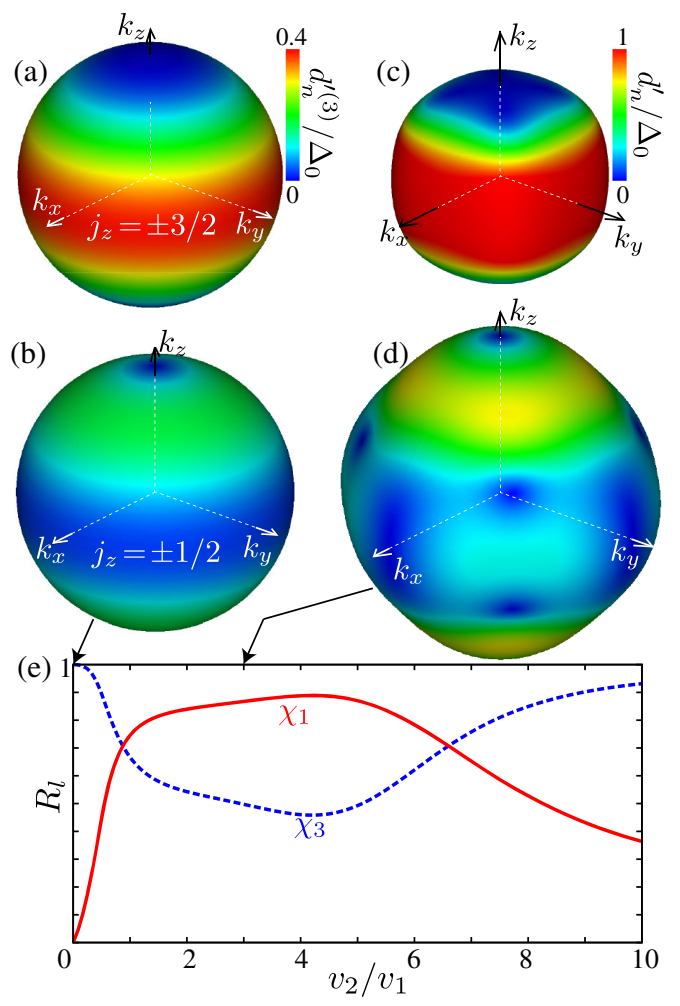

FIG. 12. Energy gap $d_{n}^{\prime}$ of $T_{1 u}$ representation on the Fermi surfaces for $v_{2}=0$ (a),(b) and $3 v_{1}$ (c),(d). (a),(c) and (b),(d) show the inner and outer Fermi surfaces, respectively. (e) Mixing ratio of angular momenta $l=1$ and $l=3$ pairs for $T_{1 u}$ gap function $\Delta=\Delta_{0}(2 / \sqrt{5})\left(\chi_{1} J_{z}+\chi_{3} \tilde{J}_{z}\right) \otimes \sigma_{y}$. The parameters are the same as in Fig. 2. 
superconductivities in terms of the gap structure. From the analytical form of wave function (27), the $J=1$ component is given as

$$
d_{j_{z}, \pm}^{(1)}(\hat{\boldsymbol{k}})= \pm \frac{2 \Delta_{0}}{\sqrt{5}} \cos \left(2 \rho_{k}\right)\left\langle j_{z}|\boldsymbol{J} \cdot \hat{\boldsymbol{k}}|-j_{z}\right\rangle,
$$

for two Fermi surfaces with the angular momentum $j_{z}=$ $\pm 3 / 2$ and $\pm 1 / 2$ along $\boldsymbol{k}$. Here, as the spin operators $J_{x}$ and $J_{y}$ shift $j_{z}$ only by \pm 1 and $J_{z}$ preserves $j_{z}$, the $J=1$ component cannot couple the $j_{z}= \pm \frac{3}{2}$ states; i.e.,

$$
d_{ \pm 3 / 2, \pm}^{\prime(1)}(\hat{\boldsymbol{k}})=0
$$

for all the directions of $\boldsymbol{k}$. In contrast, the $J=3$ component opens the energy gap in this Fermi surface, because $\tilde{J}_{x}$ and $\tilde{J}_{y}$ have finite matrix elements between $j_{z}= \pm \frac{3}{2}$ states (see Appendix A). Therefore, it is observed that the $J=3$ state is more stable than $J=1$ at this point.

Notably, the $T_{1 u}$ state realizes a nematic superconducting state, which spontaneously breaks the $O_{h}$-group symmetry. As illustrated in Figs. 12(a)-12(d), $T_{1 u}$ supports an anisotropic superconducting gap breaking the $O_{h}$ group. The nematic feature can also be used to identify the $T_{1 u}$ state through thermal transport measurements [23].

\section{CONCLUSIONS}

We have investigated topological superconductivity in doped TIs of $J=3 / 2$ electrons. Antiperovskites realize this class of topological materials, whose normal state hosts Dirac points at the topological quantum phase transition. In contrast to half-Heusler systems with a single $J=3 / 2$ band, the antiperovskites support multiple $J=3 / 2$ bands, which allows us to realize various unconventional superconductivities within the BCS type of constant gap functions. Using the $k \cdot p$ Hamiltonian, we have demonstrated that the $J=0$ odd-parity pairing state with the $A_{1 u}$ representation of $O_{h}$ group exhibits the highest transition temperature $T_{c}$ if the interorbital pairing interaction is dominant. The $A_{1 u}$ state shows a new class of topological superconductivity with a higher winding number $w_{3 \mathrm{D}}=$ \pm 2 or \pm 4 . Moreover, it displays topological crystalline superconductivity with respect to the mirror reflections and fourfold rotation of the $O_{h}$ group.

We have also revealed that the nematic $T_{1 u}$ state with $J=3$ Cooper pairs has a high $T_{c}$, which is comparable to that of $A_{1 u}$. This result suggests an interesting possibility of spin-septet nematic superconductivity in antiperovskite materials. Similar to the case of doped $\mathrm{Bi}_{2} \mathrm{Se}_{3}$, the $T_{1 u}$ phase might be stabilized against the $A_{1 u}$ phase by considering the higher-order terms of the $k \cdot p$ Hamiltonian.

\section{ACKNOWLEDGMENTS}

This work was supported by JSPS KAKENHI Grants No. JP16K17755, No. JP16H06861, No. JP17H02922, and
No. JP17J08855 and the JSPS Core-to-Core program. S. K. is supported by the CREST project (JPMJCR16F2) from Japan Science and Technology Agency (JST), and the Building of Consortia for the Development of Human Resources in Science and Technology. The numerical calculations were carried out on XC40 at YITP in Kyoto University.

\section{APPENDIX A: SPIN MATRICES IN $J=3 / 2$ SPACE}

Here, we provide the explicit forms of the matrices useful to describe $J=3 / 2$ systems. First, the spin matrices in the $J=3 / 2$ space are given by

$$
\begin{gathered}
J_{x}=\frac{1}{2}\left(\begin{array}{cccc}
0 & \sqrt{3} & 0 & 0 \\
\sqrt{3} & 0 & 2 & 0 \\
0 & 2 & 0 & \sqrt{3} \\
0 & 0 & \sqrt{3} & 0
\end{array}\right), \\
J_{y}=\frac{i}{2}\left(\begin{array}{cccc}
0 & -\sqrt{3} & 0 & 0 \\
\sqrt{3} & 0 & -2 & 0 \\
0 & 2 & 0 & -\sqrt{3} \\
0 & 0 & \sqrt{3} & 0
\end{array}\right), \\
J_{z}=\frac{1}{2}\left(\begin{array}{cccc}
3 & 0 & 0 & 0 \\
0 & 1 & 0 & 0 \\
0 & 0 & -1 & 0 \\
0 & 0 & 0 & -3
\end{array}\right) .
\end{gathered}
$$

In general, the $4 \times 4$ Hermitian matrix in the $J=3 / 2$ space is expanded by the basis given in Table I. Among them, the $T_{1}$ representation including spin matrices itself $\left\{J_{x}, J_{y}, J_{z}\right\}$ behaves as a vector under the spin rotation $C_{q n}$ defined in Eq. (1). Here, the other set of $T_{1}$ basis is given by the third-order polynomials of spin matrix, which has an explicit form as follows:

$$
\begin{aligned}
\tilde{J}_{x} & \equiv \frac{5}{3}\left(J_{y} J_{x} J_{y}+J_{z} J_{x} J_{z}\right)-\frac{7}{6} J_{x} \\
& =\frac{1}{4}\left(\begin{array}{cccc}
0 & \sqrt{3} & 0 & -5 \\
\sqrt{3} & 0 & -3 & 0 \\
0 & -3 & 0 & \sqrt{3} \\
-5 & 0 & \sqrt{3} & 0
\end{array}\right), \\
\tilde{J}_{y} & \equiv \frac{5}{3}\left(J_{z} J_{y} J_{z}+J_{x} J_{y} J_{x}\right)-\frac{7}{6} J_{y} \\
& =\frac{i}{4}\left(\begin{array}{cccc}
0 & -\sqrt{3} & 0 & -5 \\
\sqrt{3} & 0 & 3 & 0 \\
0 & -3 & 0 & -\sqrt{3} \\
5 & 0 & \sqrt{3} & 0
\end{array}\right),
\end{aligned}
$$




$$
\begin{aligned}
\tilde{J}_{z} & \equiv \frac{5}{3}\left(J_{x} J_{z} J_{x}+J_{y} J_{z} J_{y}\right)-\frac{7}{6} J_{z} \\
& =\frac{1}{2}\left(\begin{array}{cccc}
-1 & 0 & 0 & 0 \\
0 & 3 & 0 & 0 \\
0 & 0 & -3 & 0 \\
0 & 0 & 0 & 1
\end{array}\right) .
\end{aligned}
$$

By this definition, the two sets of $T_{1}$ basis satisfy the orthogonal condition

$$
\boldsymbol{J} \cdot \boldsymbol{J}=\tilde{\boldsymbol{J}} \cdot \tilde{\boldsymbol{J}}=\frac{15}{4}, \quad \boldsymbol{J} \cdot \tilde{\boldsymbol{J}}=0
$$

\section{APPENDIX B: SUPERCONDUCTIVITY WITH TIME-REVERSAL AND INVERSION SYMMETRIES}

\section{Nambu space and symmetry}

In this section, we summarize the general properties of the BdG Hamiltonian for the systems with time-reversal symmetry. Here, we use the Nambu space spanned by the basis $\left(\boldsymbol{c}_{\boldsymbol{k}}, \overline{\boldsymbol{c}}_{-\boldsymbol{k}}\right)$, where the spinor $\boldsymbol{c}_{\boldsymbol{k}}$ consists of the annihilation operator $c_{a, k}$ with the spin and orbital indices $a=\left(\sigma_{z}, j_{z}\right)$ and $\overline{\boldsymbol{c}}_{-\boldsymbol{k}}$ is its time-reversal hole partner with the components $\bar{c}_{a,-k}=\sum_{a^{\prime}}\left(C_{2, y}\right)_{a a^{\prime}} c_{a^{\prime},-k}^{\dagger}$. In this basis, the BdG Hamiltonian is written as

$$
H(\boldsymbol{k})=\left(\begin{array}{cc}
H_{0}(\boldsymbol{k}) & \Delta(\boldsymbol{k}) \\
\Delta^{\dagger}(\boldsymbol{k}) & -H_{0}(\boldsymbol{k})
\end{array}\right),
$$

where we have used the time-reversal symmetry of the oneparticle Hamiltonian in the normal state (3).

The point-group operation for the particle space is given as $c_{a k} \rightarrow \sum_{a} G_{a a^{\prime}} c_{a^{\prime}, k}$ with $G=C_{q, n}, P$, or $P C_{q, n}$ corresponding to discrete rotation, inversion, and improper rotation acting on the internal d.o.f. $a=\left(\sigma_{z}, j_{z}\right)$, respectively. The transformation $G$ for the time-reversal hole is $\quad \bar{c}_{a,-\boldsymbol{k}} \rightarrow \sum_{a^{\prime}, b^{\prime}}\left(C_{2, \boldsymbol{y}}\right)_{a a^{\prime}}\left(\mathcal{K} G_{a^{\prime} b^{\prime}} \mathcal{K}^{-1}\right) c_{b^{\prime},-\boldsymbol{k}}^{\dagger}=$ $\sum_{a^{\prime}, b^{\prime}} G_{a, a^{\prime}}\left(C_{2, y}\right)_{a^{\prime} b^{\prime}} c_{b^{\prime},-k}^{\dagger}=\sum_{a^{\prime}} G_{a a^{\prime}} \bar{c}_{a^{\prime},-k}$, because the time-reversal operator $\mathcal{T}=C_{2, y} \mathcal{K}$ commutes with any point-group operation $G$. The transformations for the particle and time-reversal hole components are similar. $\mathcal{T}$ and $G$ act on the gap function as

$$
\mathcal{T} \Delta(-\boldsymbol{k}) \mathcal{T}^{-1} \quad \text { and } \quad G \Delta\left(D_{G}^{-1}[\boldsymbol{k}]\right) G^{-1},
$$

where $D_{G}[\boldsymbol{k}]$ indicates the $G$ operation of $\boldsymbol{k}$. When $\mathcal{T} \Delta(-\boldsymbol{k}) \mathcal{T}^{-1}=\Delta(\boldsymbol{k})$, the BdG Hamiltonian is time-reversal invariant:

$$
\tilde{\mathcal{T}} H(\boldsymbol{k}) \tilde{\mathcal{T}}^{-1}=H(-\boldsymbol{k}) \quad \text { with } \quad \tilde{\mathcal{T}}=\mathcal{T} \tau_{0} .
$$

The Fermi statistics of $\boldsymbol{c}_{\boldsymbol{k}}$ result in a restriction on the gap function

$$
\left[\Delta(\boldsymbol{k}) C_{2, y}\right]^{T}=-\Delta(-\boldsymbol{k}) C_{2, y}
$$

To satisfy both Eqs. (B3) and (B4), the gap function is Hermitian:

$$
\Delta^{\dagger}(\boldsymbol{k})=\Delta(\boldsymbol{k})
$$

Consequently, the BdG Hamiltonian (B1) for the timereversal symmetric superconductivity is reduced to

$$
H(\boldsymbol{k})=H_{0}(\boldsymbol{k}) \tau_{z}+\Delta(\boldsymbol{k}) \tau_{x},
$$

where $\tau_{i}$ represents the Pauli matrices acting on the particle and time-reversal hole space.

We also consider the particle-hole symmetry of the BdG Hamiltonian

$\mathcal{C} H(\boldsymbol{k}) \mathcal{C}^{-1}=-H(-\boldsymbol{k}) \quad$ with $\quad \mathcal{C}=\left(\begin{array}{cc}0 & C_{2, y}^{\dagger} \\ C_{2, y} & 0\end{array}\right) \mathcal{K}$,

inherent to superconductors. By combining Eqs. (B7) and (B3), chiral symmetry is given as

$$
\Gamma H(\boldsymbol{k}) \Gamma^{-1}=-H(\boldsymbol{k}) \quad \text { with } \quad \Gamma=-i \tilde{\mathcal{T}} \mathcal{C}=\tau_{y} .
$$

\section{Band representation}

In this section, we consider the BdG Hamiltonian in terms of the band basis of the normal state. This basis is given by the Bloch equation of the normal state

$$
H_{0}(\boldsymbol{k})\left|u_{n, \boldsymbol{k}}\right\rangle=\xi_{n}(\boldsymbol{k})\left|u_{n, \boldsymbol{k}}\right\rangle
$$

with the band index $n$ and eigenenergy $\xi_{n}(\boldsymbol{k})$. From orthonormality and completeness of basis,

$$
\left\langle u_{n, k} \mid u_{n^{\prime}, k}\right\rangle=\delta_{n n^{\prime}}, \quad \sum_{n}\left|u_{n, k}\right\rangle\left\langle u_{n, k}\right|=\hat{1},
$$

the one-particle Hamiltonian is written as a diagonal form on this basis:

$$
H_{0}(\boldsymbol{k})=\sum_{n} \xi_{n}(\boldsymbol{k})\left|u_{n, \boldsymbol{k}}\right\rangle\left\langle u_{n, \boldsymbol{k}}\right|
$$

For later convenience, we consider the time-reversal and inversion symmetry of the basis. From Eq. (3) for the oneparticle Hamiltonian of the normal state, the time-reversal partner of $\left|u_{n, k}\right\rangle$ is

$$
\left|u_{\bar{n},-k}\right\rangle=\mathcal{T}\left|u_{n, k}\right\rangle,
$$

with $\bar{n} \neq n$ because of $\mathcal{T}^{2}=-1$. From Eq. (2), the inversion partner is 


$$
\left|u_{n,-k}\right\rangle=P\left|u_{n, k}\right\rangle .
$$

In the presence of both symmetries, the energy of the state

$$
\left|u_{\bar{n}, k}\right\rangle=P \mathcal{T}\left|u_{n, k}\right\rangle
$$

is degenerated with that of $\left|u_{n, k}\right\rangle$; i.e., $\xi_{\bar{n}}=\xi_{n}$.

Let us consider the band representation of the gap function. As both $\left|u_{n, k}\right\rangle$ and $\left|u_{\bar{n}, k}\right\rangle$ have completeness (B10), we can expand the gap function as

$$
\Delta(\boldsymbol{k})=-\sum_{n, n^{\prime}} d_{n, n^{\prime}}(\boldsymbol{k})\left|u_{n, \boldsymbol{k}}\right\rangle\left\langle u_{\bar{n}^{\prime}, \boldsymbol{k}}\right|
$$

with the matrix element $d_{n, n^{\prime}}=-\left\langle u_{n, \boldsymbol{k}}|\Delta(\boldsymbol{k})| u_{\bar{n}^{\prime}, \boldsymbol{k}}\right\rangle=$ $-\left\langle u_{n, k}|\Delta \mathcal{T}| u_{n^{\prime},-k}\right\rangle$. The $d_{n, n^{\prime}}$ provides the superconducting correlation for Cooper pairs consisting of $\left|u_{n, k}\right\rangle$ and $\left|u_{n^{\prime},-k}\right\rangle$. In the weak-coupling limit where the gap function $\Delta$ is much smaller than the level spacing among the different bands, the matrix element $d_{n, n^{\prime}}$ is nonzero only for the degenerated bands $n^{\prime}=n$ or $n^{\prime}=\bar{n}$ [see Eq. (B14)]. This result indicates that the time-reversal or inversion partners can form the Cooper pair. These pairing states are described by the matrix elements

$$
d_{n, n^{\prime}}= \begin{cases}d_{n} \delta_{n, \bar{n}^{\prime}} & \text { for time-reversal pair } \\ d_{n}^{\prime} \delta_{n, n^{\prime}} & \text { for inversion pair. }\end{cases}
$$

In particular, when the time-reversal partners form the Cooper pair, not only the normal Hamiltonian (B11) but also the gap function is diagonal in the band representation [see Eq. (B16)]. Therefore, the BdG Hamiltonian is also diagonal:

$$
H(\boldsymbol{k})=\sum_{n}\left[\xi_{n}(\boldsymbol{k}) \tau_{z}-d_{n}(\boldsymbol{k}) \tau_{x}\right]\left|u_{n, \boldsymbol{k}}\right\rangle\left\langle u_{n, \boldsymbol{k}}\right|,
$$

where $d_{n}=\left\langle u_{n, k}|\Delta| u_{n, k}\right\rangle$ is real owing to Hermiticity (B5). Note that the time-reversal and inversion symmetries for the gap function are

$$
\begin{gathered}
d_{\bar{n}}(-\boldsymbol{k})=d_{n}(\boldsymbol{k}) \\
d_{n}(-\boldsymbol{k})= \pm d_{n}(\boldsymbol{k}),
\end{gathered}
$$

respectively, where the double sign indicates even- and odd-parity superconductivities.

\section{3D winding number for time-reversal pairs}

The time-reversal and particle-hole symmetries defined by Eqs. (B3) and (B7) do not depend on any particular crystal structure. They thus specify the most general symmetry-protected topological number [14]. In the present system with $\mathcal{T}^{2}=-1$ and $\mathcal{C}^{2}=+1$, the general topological number is the $3 \mathrm{D}$ winding number

$$
w_{3 \mathrm{D}}=\int \frac{d^{3} k}{48 \pi^{2}} \epsilon^{\alpha \beta \gamma} \operatorname{Tr}\left[\Gamma\left(H^{-1} \partial_{\alpha} H\right)\left(H^{-1} \partial_{\beta} H\right)\left(H^{-1} \partial_{\gamma} H\right)\right]
$$

with the 3D Levi-Civita symbol $\epsilon^{\alpha \beta \gamma}$. For the time-reversal pairing state, the diagonal form of the Hamiltonian (B17) provides a simple analytic form of $w_{3 \mathrm{D}}$. Here, we derive it by applying the procedure in Ref. [62] to the system with the additional inversion symmetry.

First, we consider the unitary transformation $\left(\tau_{x}, \tau_{y}, \tau_{z}\right) \rightarrow$ $\left(\tau_{y}, \tau_{z}, \tau_{x}\right)$. It yields the eigenbasis of the chiral operator with $\Gamma=\tau_{z}$, convenient to analyze $w_{3 \mathrm{D}}$. In this basis, the $\mathrm{BdG}$ Hamiltonian (B17) is written as

$$
\begin{aligned}
H(\boldsymbol{k}) & =\sum_{n}\left[\xi_{n}(\boldsymbol{k}) \tau_{x}-d_{n}(\boldsymbol{k}) \tau_{y}\right]\left|u_{n, \boldsymbol{k}}\right\rangle\left\langle u_{n, \boldsymbol{k}}\right| \\
& =\left(\begin{array}{cc}
0 & h(\boldsymbol{k}) \\
h^{\dagger}(\boldsymbol{k}) & 0
\end{array}\right),
\end{aligned}
$$

where the off-diagonal element is

$$
h(\boldsymbol{k})=\sum_{n}\left[\xi_{n}(\boldsymbol{k})+i d_{n}(\boldsymbol{k})\right]\left|u_{n, \boldsymbol{k}}\right\rangle\left\langle u_{n, \boldsymbol{k}}\right| .
$$

Second, we use the so-called spectral flattening technique [10]. The eigenenergy of Eq. (B21) is given by the absolute values of the off-diagonal elements $E=$ $\pm\left|\xi_{n}+i d_{n}\right|$. Therefore, the replacement

$$
\begin{aligned}
h(\boldsymbol{k}) \rightarrow q(\boldsymbol{k}) & =\sum_{n} \frac{\xi_{n}(\boldsymbol{k})+i d_{n}(\boldsymbol{k})}{\left|\xi_{n}(\boldsymbol{k})+i d_{n}(\boldsymbol{k})\right|}\left|u_{n, \boldsymbol{k}}\right\rangle\left\langle u_{n, \boldsymbol{k}}\right| \\
& =\sum_{n} e^{i \vartheta_{n}(\boldsymbol{k})}\left|u_{n, \boldsymbol{k}}\right\rangle\left\langle u_{n, \boldsymbol{k}}\right|
\end{aligned}
$$

provides the adiabatic transformation of the original Hamiltonian to that with flat bands with $E= \pm 1$ without changing $w_{3 \mathrm{D}}$. The phase factor $\vartheta_{n}(\boldsymbol{k})$ is defined as in Fig. 13. In this technique, the Hamiltonian is replaced as

$$
H \rightarrow Q(\boldsymbol{k})=\left(\begin{array}{cc}
0 & q(\boldsymbol{k}) \\
q^{\dagger}(\boldsymbol{k}) & 0
\end{array}\right),
$$

and its inverse is replaced as

$$
H^{-1} \rightarrow Q^{-1}(\boldsymbol{k})=Q(\boldsymbol{k}),
$$

where we have used $q^{-1}(\boldsymbol{k})=q^{\dagger}(\boldsymbol{k})$. Through this replacement, we can evaluate the 3D winding number (B20) as 


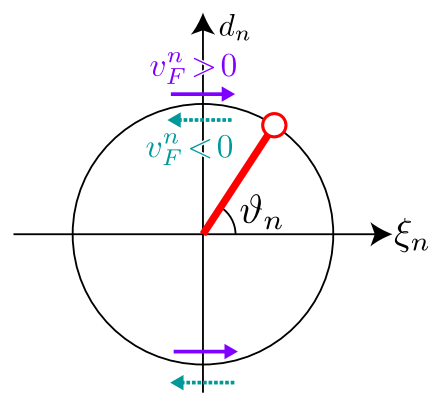

FIG. 13. Phase factor $\vartheta_{n}(k)$ and its path with the increase in $k$.

$$
\begin{aligned}
w_{3 \mathrm{D}}= & \int \frac{d^{3} k}{48 \pi^{2}} \epsilon^{\alpha \beta \gamma} \operatorname{Tr}\left[\Gamma\left(Q \partial_{\alpha} Q\right)\left(Q \partial_{\beta} Q\right)\left(Q \partial_{\gamma} Q\right)\right] \\
= & \int \frac{d^{3} k}{24 \pi^{2}} \epsilon^{\alpha \beta \gamma} \operatorname{Tr}\left[\left(q \partial_{\alpha} q^{\dagger}\right)\left(q \partial_{\beta} q^{\dagger}\right)\left(q \partial_{\gamma} q^{\dagger}\right)\right] \\
= & \int \frac{d^{3} k}{4 \pi^{2}} \epsilon_{\alpha \beta \gamma} \sum_{n, n^{\prime}}\left[\left(i \partial_{\gamma} \vartheta_{n}\right)\left\langle\partial_{\alpha} u_{n, \boldsymbol{k}} \mid \partial_{\beta} u_{n, \boldsymbol{k}}\right\rangle \delta_{n, n^{\prime}}\right. \\
& -\left(i \partial_{\gamma} \vartheta_{n}\right) \cos \vartheta_{n^{\prime} n}\left\langle\partial_{\alpha} u_{n, \boldsymbol{k}} \mid u_{n^{\prime}, \boldsymbol{k}}\right\rangle\left\langle u_{n^{\prime}, \boldsymbol{k}} \mid \partial_{\beta} u_{n, \boldsymbol{k}}\right\rangle \\
& \left.-i \sin \vartheta_{n^{\prime} n}\left\langle\partial_{\alpha} u_{n^{\prime}, \boldsymbol{k}} \mid \partial_{\beta} u_{n, \boldsymbol{k}}\right\rangle\left\langle\partial_{\gamma} u_{n, \boldsymbol{k}} \mid u_{n^{\prime}, \boldsymbol{k}}\right\rangle\right]
\end{aligned}
$$

with $\vartheta_{n^{\prime} n}=\vartheta_{n^{\prime}}-\vartheta_{n}$. To obtain the last equality in Eq. (B26), we use the time-reversal symmetry for the phase $\vartheta_{\bar{n}}(-\boldsymbol{k})=\vartheta_{n}(\boldsymbol{k})$ given by Eq. (B18) and for the eigenstates $\left\langle\partial_{\alpha} u_{\bar{n},-k} \mid \partial_{\beta} u_{\bar{n}^{\prime},-k}\right\rangle=\left\langle\partial_{\beta} u_{n^{\prime}, k} \mid \partial_{\alpha} u_{n, k}\right\rangle$ and $\left\langle\partial_{\alpha} u_{\bar{n},-k} \mid u_{\bar{n}^{\prime},-k}\right\rangle=\left\langle u_{n^{\prime}, k} \mid \partial_{\alpha} u_{n, k}\right\rangle$ by Eq. (B14) in addition to the usual algebraic expansion.

Subsequently, we use the weak-coupling limit with $d_{n} \rightarrow 0$, where the phase factor is given by the step function

$$
\vartheta_{n}(\boldsymbol{k})= \begin{cases}0 & \text { for } k>k_{F}, \\ \pi & \text { for } k<k_{F}, \\ \pi / 2 & \text { for } k=k_{F} \text { and } d_{n}>0 \\ -\pi / 2 & \text { for } k=k_{F} \text { and } d_{n}<0\end{cases}
$$

The contribution from the third term in Eq. (B26) is zero in this limit. In addition, away from the Fermi surface, the derivative of the phase factor $\partial_{\gamma} \vartheta_{n}=0$.

Let us consider the nontrivial contribution for the $3 \mathrm{D}$ winding number in Eq. (B26) from momenta close to the Fermi surface. Here, we consider the coordinate in momentum space $\left(k_{\alpha}, k_{\beta}, k_{\gamma}\right)$ as $\left(k, k_{1}^{\prime}, k_{2}^{\prime}\right)$ with momenta $k$ and $k_{\alpha=1,2}^{\prime}$ perpendicular and parallel to the Fermi surface, respectively. The weak-coupling limit (B27) yields $\partial_{k_{\alpha}^{\prime}} \vartheta_{n}=0$. In addition, the derivative with respect to $k$ is

$$
\partial_{k} \vartheta_{n}=-\operatorname{sgn}\left[v_{F}^{n} \Delta_{n}\right] \pi \delta\left(k-k_{F}\right)
$$

where $v_{F}^{n}$ is the Fermi velocity of band $n$. Consequently, the $3 \mathrm{D}$ winding number (B26) is reduced to

$$
\begin{aligned}
w_{3 \mathrm{D}}= & -i \operatorname{sgn}\left[v_{F^{n}} \Delta_{n}\right] \sum_{n} \int \frac{d^{2} k^{\prime}}{4 \pi} \epsilon_{\alpha \beta}\left[\left\langle\partial_{k_{\alpha}^{\prime}} u_{n, k} \mid \partial_{k_{\beta}^{\prime}} u_{n, k}\right\rangle\right. \\
& \left.-\sum_{n^{\prime}} \cos \vartheta_{n^{\prime} n}\left(k_{F}^{n}\right)\left\langle\partial_{k_{\alpha}^{\prime}} u_{n, k} \mid u_{n^{\prime}, k}\right\rangle\left\langle u_{n^{\prime}, k} \mid \partial_{k_{\beta}^{\prime}} u_{n, k}\right\rangle\right] .
\end{aligned}
$$

Equation (B27) and the time-reversal and inversion symmetries $\vartheta_{\bar{n}}(\boldsymbol{k})= \pm \vartheta_{n}(\boldsymbol{k})$ yield the phase factor at the Fermi surface as

$$
\cos \left[\vartheta_{n}\left(k_{F}^{n}\right)-\vartheta_{n^{\prime}}\left(k_{F}^{n}\right)\right]= \begin{cases}1 & \text { for } n^{\prime}=n \\ \pm 1 & \text { for } n^{\prime}=\bar{n} \\ 0 & \text { otherwise }\end{cases}
$$

where the double sign indicates even- and odd-parity superconductivities. When $n^{\prime}=n$, the second term in Eq. (B29) is zero owing to $\epsilon_{\alpha \beta}\left\langle\partial_{k_{\alpha}^{\prime}} u_{n, k} \mid u_{n, k}\right\rangle \times$ $\left\langle u_{n, k} \mid \partial_{k_{\beta}^{\prime}} u_{n, k}\right\rangle=0$. In addition, even for $n^{\prime}=\bar{n}$, the second term in Eq. (B29) is

$$
\begin{aligned}
f_{n} & =\epsilon_{\alpha \beta}\left\langle\partial_{k_{\alpha}^{\prime}} u_{n, \boldsymbol{k}} \mid u_{\bar{n}, \boldsymbol{k}}\right\rangle\left\langle u_{\bar{n}, \boldsymbol{k}} \mid \partial_{k_{\beta}^{\prime}} u_{n, \boldsymbol{k}}\right\rangle \\
& =-\epsilon_{\alpha \beta}\left\langle\partial_{k_{\alpha}^{\prime}} u_{\bar{n}, \boldsymbol{k}} \mid u_{n, \boldsymbol{k}}\right\rangle\left\langle u_{n, \boldsymbol{k}} \mid \partial_{k_{\beta}^{\prime}} u_{\bar{n}, \boldsymbol{k}}\right\rangle=-f_{\bar{n}},
\end{aligned}
$$

where we use $\epsilon_{\alpha \beta}=-\epsilon_{\beta \alpha}$ and $\left\langle\partial_{\alpha} u_{n, k} \mid u_{n^{\prime}, k}\right\rangle=$ $-\left\langle u_{n, k} \mid \partial_{\alpha} u_{n^{\prime}, k}\right\rangle$. Therefore, the contribution from $f_{\bar{n}}$ cancels that from $f_{n}$ when performing a summation over $n$ and $\bar{n}$. Consequently, only the first term in Eq. (B29) yields the nontrivial contribution to $w_{3 \mathrm{D}}$.

Consequently, the 3D winding number is given by

$$
w_{3 \mathrm{D}}=\frac{1}{2} \sum_{n} \operatorname{sgn}\left[v_{F} d_{n}\right] \nu_{\mathrm{Ch}}^{n}
$$

with the first Chern number

$$
\nu_{\mathrm{Ch}}^{n}=-\frac{i}{2 \pi} \int_{k_{F}} d^{2} k^{\prime} \epsilon^{\alpha \beta}\left\langle\partial_{k_{\alpha}^{\prime}} u_{n, k} \mid \partial_{k_{\beta}^{\prime}} u_{n, \boldsymbol{k}}\right\rangle
$$

\section{Linearized gap equation}

In this section, we derive the linearized gap equation for the system with time-reversal and inversion symmetries. The mean-field Hamiltonian of the superconducting system is given as

$$
\hat{H}(\boldsymbol{k})=\left(\begin{array}{cc}
\boldsymbol{c}_{k}^{\dagger} & \overline{\boldsymbol{c}}_{-k}^{\dagger}
\end{array}\right) H(\boldsymbol{k})\left(\begin{array}{c}
\boldsymbol{c}_{\boldsymbol{k}} \\
\overline{\boldsymbol{c}}_{-\boldsymbol{k}}
\end{array}\right)
$$

where the spinor $\boldsymbol{c}_{\boldsymbol{k}}$ consists of the annihilation operator $c_{j_{z}, \sigma_{z}, k}$ with the indices $j_{z}$ and $\sigma_{z}$ for spin and orbital, respectively, its time-reversal hole partner $\overline{\boldsymbol{c}}_{-\boldsymbol{k}}$ is given as $\bar{c}_{j_{z}, \sigma_{z},-\boldsymbol{k}}=\sum_{j_{z}^{\prime}}\left(C_{2, \hat{y}^{\prime}}\right)_{j_{z} j_{z}^{\prime}} c_{j_{z}^{\prime}, \sigma_{z},-\boldsymbol{k}}^{\dagger}$, and $H(\boldsymbol{k})$ is the BdG 
Hamiltonian given in Eq. (B1). Here, we define the thermal and anomalous Green's functions

$$
\begin{gathered}
\mathcal{G}_{\boldsymbol{k}}\left(\tau, \tau^{\prime}\right)=-\left\langle T_{\tau}\left[\boldsymbol{c}_{\boldsymbol{k}}(\tau) \boldsymbol{c}_{\boldsymbol{k}}^{\dagger}\left(\tau^{\prime}\right)\right]\right\rangle, \\
\mathcal{F}_{\boldsymbol{k}}\left(\tau, \tau^{\prime}\right)=-\left\langle T_{\tau}\left[\overline{\boldsymbol{c}}_{-\boldsymbol{k}}(\tau) \boldsymbol{c}_{\boldsymbol{k}}^{\dagger}\left(\tau^{\prime}\right)\right]\right\rangle,
\end{gathered}
$$

where $\boldsymbol{c}_{\boldsymbol{k}}(\tau)=e^{H_{0} \tau / \hbar} \boldsymbol{c}_{\boldsymbol{k}} e^{-H_{0} \tau / \hbar}$ is the Heisenberg operator for imaginary time $\tau$. From the Heisenberg equation of $\boldsymbol{c}_{\boldsymbol{k}}(\tau)$, we obtain the Gor'kov equation of the Green's function [76]:

$$
\begin{array}{r}
{\left[i \hbar \omega_{N}-H_{0}(\boldsymbol{k})\right] \mathcal{G}_{\boldsymbol{k}}\left(\omega_{N}\right)-\Delta \mathcal{F}_{\boldsymbol{k}}\left(\omega_{N}\right)=\hbar,} \\
{\left[i \hbar \omega_{N}+H_{0}(\boldsymbol{k})\right] \mathcal{F}_{\boldsymbol{k}}\left(\omega_{N}\right)-\Delta \mathcal{G}_{\boldsymbol{k}}\left(\omega_{N}\right)=0,}
\end{array}
$$

where we use the time-reversal symmetry of the normal state (3) and of the superconducting gap function (B5). The Fourier component of thermal and anomalous Green's functions is given by

$$
\mathcal{G}_{k}\left(\tau, \tau^{\prime}\right)=\frac{1}{\beta \hbar} \sum_{N} e^{i \omega_{N}\left(\tau-\tau^{\prime}\right)} \mathcal{G}_{k}\left(\omega_{N}\right)
$$

with fermionic Matsubara frequency $\omega_{N}=(2 N+1) \pi / \hbar \beta$ and $\beta=1 / k_{B} T$. In terms of the Matsubara anomalous Green's function, the gap equation is written as

$$
\Delta=-\lim _{\eta \rightarrow 0} \frac{1}{\hbar \beta} \sum_{N} \int d^{3} k \tilde{V} e^{i \omega_{N} \eta} \mathcal{F}_{\boldsymbol{k}}\left(\omega_{N}\right),
$$

where the interaction is $\tilde{V}=U(V)$ when the gap function $\Delta$ is intended for intraorbital (interorbital) coupling [see also Eq. (16)].

Near the $T_{c}$ where $\Delta \rightarrow 0$, we can linearize the gap equation. By solving Eq. (B37) for $\mathcal{G}_{\boldsymbol{k}}$ and $\mathcal{F}_{\boldsymbol{k}}$ and substituting them successively, we approximate the anomalous Green's function in the lowest order of $\Delta$ as

$$
\mathcal{F}_{\boldsymbol{k}}\left(\omega_{N}\right)=-\frac{1}{\hbar} \mathcal{G}_{\boldsymbol{k}}^{0}\left(-\omega_{N}\right) \Delta \mathcal{G}_{\boldsymbol{k}}^{0}\left(\omega_{N}\right)+\mathcal{O}\left(\Delta^{2}\right),
$$

with the single-particle Green's function $\mathcal{G}_{\boldsymbol{k}}^{0}\left(\omega_{N}\right)=$ $\hbar\left[i \hbar \omega_{N}-H_{0}(\boldsymbol{k})\right]^{-1}$. It is written in terms of the band representation (B11) as

$$
\mathcal{G}_{\boldsymbol{k}}^{0}\left(\omega_{N}\right)=\sum_{n} \frac{\hbar\left|u_{n, k}\right\rangle\left\langle u_{n, k}\right|}{i \hbar \omega_{N}-\xi_{n}(\boldsymbol{k})}
$$

By using Eqs. (B40) and (B41), we can linearize the gap equation (B39) as

$$
\begin{aligned}
\Delta= & -\lim _{\eta \rightarrow 0} \frac{1}{\beta} \sum_{N, n, n^{\prime}} \int d^{3} k \tilde{V} e^{i \omega_{N} \eta} \\
& \times \frac{\left|u_{n, \boldsymbol{k}}\right\rangle\left\langle u_{n, \boldsymbol{k}}|\Delta| u_{n^{\prime}, \boldsymbol{k}}\right\rangle\left\langle u_{n^{\prime}, \boldsymbol{k}}\right|}{\left(-i \hbar \omega_{N}-\xi_{n}\right)\left(i \hbar \omega_{N}-\xi_{n^{\prime}}\right)} .
\end{aligned}
$$

In addition, the matrix element $d_{n, n^{\prime}}=\left\langle u_{n, \boldsymbol{k}}|\Delta| u_{n^{\prime}, k}\right\rangle$ is nonzero for $n^{\prime}=n$ or $\bar{n}$ [see also Eq. (B16)], as the timereversal or inversion partners form the Cooper pair. Hence, we reduce Eq. (B42) as

$$
\begin{aligned}
\Delta= & -\lim _{\eta \rightarrow 0} \frac{1}{\beta} \sum_{N, n} \int d^{3} k \tilde{V} e^{i \omega_{N} \eta} \\
& \times \sum_{n^{\prime}=n, \bar{n}} \frac{\left|u_{n, \boldsymbol{k}}\right\rangle\left\langle u_{n, \boldsymbol{k}}|\Delta| u_{n^{\prime}, \boldsymbol{k}}\right\rangle\left\langle u_{n^{\prime}, \boldsymbol{k}}\right|}{\hbar^{2} \omega_{N}^{2}-\xi_{n}^{2}} \\
= & -\sum_{n} \int d \xi_{n} d^{2} k^{\prime} D_{n}(\boldsymbol{k}) \tilde{V} \frac{1}{4 \xi_{n}} \tanh \frac{\beta \xi_{n}}{2} \\
& \times \sum_{n^{\prime}=n, \bar{n}}\left|u_{n, \boldsymbol{k}}\right\rangle\left\langle u_{n, \boldsymbol{k}}|\Delta| u_{n^{\prime}, \boldsymbol{k}}\right\rangle\left\langle u_{n^{\prime}, \boldsymbol{k}}\right|,
\end{aligned}
$$

where we use $\xi_{\bar{n}}=\xi_{n}$, the expansion formula $z^{-1} \tanh (z / 2)=4 \sum_{n=0}^{\infty}\left[(2 n+1)^{2} \pi^{2}+z^{2}\right]^{-1}$, and the momentum coordinates $\left(k, k_{1}^{\prime}, k_{2}^{\prime}\right)$ with momenta $k$ and $k_{1,2}^{\prime}$ perpendicular and parallel to the Fermi surface, respectively. $D_{n}(\boldsymbol{k})=\left[(d k) /\left(d \xi_{n}\right)\right]$ is the density of states.

In the weak-coupling limit where $\beta_{c}^{-1}=k_{B} T_{c}$ is much smaller than any other energy scale, the factor $\xi_{n}^{-1} \tanh \left(\beta_{c} \xi_{n} / 2\right)$ has a sharp peak around the Fermi level $\xi_{n}=0$. Hence, we can approximate that the other factors are represented by their values at the Fermi surface $\xi_{n}=0$. Therefore, Eq. (B43) is rewritten as

$$
\begin{aligned}
\Delta= & -\sum_{n} \frac{I(\beta)}{2} \int_{k_{F}} d^{2} k^{\prime} D_{n}\left(\boldsymbol{k}^{\prime}\right) \tilde{V} \\
& \times \sum_{n^{\prime}=n, \bar{n}}\left|u_{n, \boldsymbol{k}^{\prime}}\right\rangle\left\langle u_{n, \boldsymbol{k}^{\prime}}|\Delta| u_{n^{\prime}, \boldsymbol{k}^{\prime}}\right\rangle\left\langle u_{n^{\prime}, \boldsymbol{k}^{\prime}}\right|,
\end{aligned}
$$

where the energy integral is evaluated as $I(\beta)=$ $\int_{-\hbar \omega_{0}}^{\hbar \omega_{0}} d \xi[1 /(2 \xi)] \tanh [(\beta \xi) / 2]=\ln \left(2 e^{\gamma} \beta \hbar \omega_{0} / \pi\right) \quad$ with Euler's constant $\gamma$ and the cutoff frequency $\omega_{0}$ [76].

In the system discussed in the main text, the gap function is expanded as $\Delta=\sum_{\alpha} \Delta_{\alpha} \Phi_{\alpha}$ [see Eq. (14)]. By using the orthonormality of the basis $\operatorname{tr}\left[\Phi_{\alpha} \Phi_{\alpha^{\prime}}\right]=\delta_{\alpha \alpha^{\prime}}$ in Eq. (B44), we obtain the gap equation for $\Delta_{\alpha}$ as

$$
\sum_{\beta} X_{\alpha \alpha^{\prime}}(\beta) \Delta_{\alpha^{\prime}}=0
$$

with the coefficient 


$$
\begin{aligned}
X_{\alpha \alpha^{\prime}}(\beta)= & \delta_{\alpha \alpha^{\prime}}+\sum_{n} \frac{I(\beta)}{2} \int_{k_{F}} d^{2} k^{\prime} D_{n}\left(\boldsymbol{k}^{\prime}\right) \tilde{V} \\
& \times \sum_{n^{\prime}=n, \bar{n}}\left\langle u_{n^{\prime}, \boldsymbol{k}^{\prime}}\left|\Phi_{\alpha}\right| u_{n, \boldsymbol{k}^{\prime}}\right\rangle\left\langle u_{n, k^{\prime}}\left|\Phi_{\alpha^{\prime}}\right| u_{n^{\prime}, \boldsymbol{k}^{\prime}}\right\rangle .
\end{aligned}
$$

The transition temperature is given by $\operatorname{det} X\left(\beta_{c}\right)=0$.

\section{APPENDIX C: DUALITY RELATION FOR $k \cdot p$ HAMILTONIAN}

It can be easily verified that the identical vectors $\boldsymbol{J}$ and $\tilde{\boldsymbol{J}}$ in the spin space hold that

$$
\begin{aligned}
& D_{J} J_{i} D_{J}^{\dagger}=-\frac{3}{5} J_{i}-\frac{4}{5} \tilde{J}_{i}, \\
& D_{J} \tilde{J}_{i} D_{J}^{\dagger}=-\frac{4}{5} J_{i}+\frac{3}{5} \tilde{J}_{i},
\end{aligned}
$$

with the unitary matrix acting on the $\operatorname{spin} J=3 / 2$ space

$$
D_{J}=\left(\begin{array}{cccc}
0 & 0 & 1 & 0 \\
0 & 0 & 0 & -1 \\
-1 & 0 & 0 & 0 \\
0 & 1 & 0 & 0
\end{array}\right) .
$$

As the $A_{1 u}$ gap function is invariant under $D_{J}$, this unitary transformation maps the system with a set of parameters $v_{1}$ and $v_{2}$ to that with another set:

$$
\left(\begin{array}{c}
v_{1} \\
v_{2}
\end{array}\right) \stackrel{D_{J}}{\rightarrow}\left(\begin{array}{c}
v_{1}^{\prime} \\
v_{2}^{\prime}
\end{array}\right)=\left(\begin{array}{l}
-\left[3 v_{1}+4 v_{2}\right] / 5 \\
-\left[4 v_{1}-3 v_{2}\right] / 5
\end{array}\right)
$$

which yields the "duality" of the BdG Hamiltonian. In addition, the operator $D_{J}$ commutes with the chiral operator

$$
\left[\Gamma, D_{J}\right]=0 .
$$

Therefore, the systems at the two sets of parameters in Eq. (C4) yield the same winding number $w_{3 \mathrm{D}}$.

Similarly, the inversion operator for the superconducting state maps the parameters as

$$
\left(\begin{array}{l}
v_{1} \\
v_{2}
\end{array}\right) \stackrel{\tilde{P}}{\rightarrow}\left(\begin{array}{c}
v_{1}^{\prime} \\
v_{2}^{\prime}
\end{array}\right)=\left(\begin{array}{l}
-v_{1} \\
-v_{2}
\end{array}\right) \quad \text { with } \quad \tilde{P}=\sigma_{z} \tau_{z} .
$$

Note that this mapping does not change $v_{2} / v_{1}$. As the operator $\tilde{P}$ anticommutes with the chiral operator $\Gamma$,

$$
\{\Gamma, \tilde{P}\}=0,
$$

the systems at the two sets of parameters in Eq. (C6) yield the winding number $w_{3 \mathrm{D}}$ with the opposite sign.

Finally, in Fig. 14, we summarize these relations in the parameter space spanned by $v_{1}$ and $v_{2} / v_{1}$ used in the main

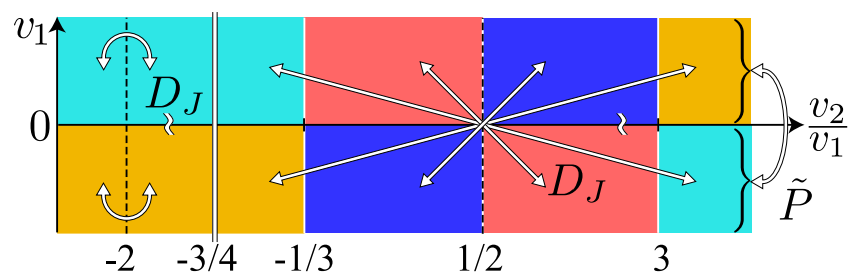

FIG. 14. Relations between the systems with parameters $v_{1}$ and $v_{2} / v_{1}$ mapped using Eqs. (C4) and (C6). The color code indicates the winding number $w_{3 \mathrm{D}}=-2$ (turquoise), 4 (red), -4 (blue), and 2 (yellow), the same as in Fig. 10.

text. For $v_{2} / v_{1}>-3 / 4$, the unitary transformation $D_{J}$ maps $v_{2} / v_{1}$ to the opposite side of $v_{2} / v_{1}=1 / 2$ denoted by the dashed line in Fig. 14. Simultaneously, it changes the sign of $v_{1}$. Furthermore, we can map a system with $v_{1}$ to another with $-v_{1}$ using $\tilde{P}$. Therefore, at the opposite side with respect to $v_{2} / v_{1}=1 / 2$ and with the same sign as $v_{1}$, the winding number $w_{3 \mathrm{D}}$ exhibits the opposite sign. In contrast, for $v_{2} / v_{1}<-3 / 4$, the unitary transformation $D_{J}$ connects the parameters with the same sign as $v_{1}$ (see Fig. 14), which have the same winding number $w_{3 \mathrm{D}}$.

[1] M. Z. Hasan and C.L. Kane, Colloquium: Topological Insulators, Rev. Mod. Phys. 82, 3045 (2010).

[2] X.-L. Qi and S.-C. Zhang, Topological Insulators and Superconductors, Rev. Mod. Phys. 83, 1057 (2011).

[3] Y. Tanaka, M. Sato, and N. Nagaosa, Symmetry and Topology in Superconductors-Odd-Frequency Pairing and Edge States, J. Phys. Soc. Jpn. 81, 011013 (2012).

[4] Y. Ando, Topological Insulator Materials, J. Phys. Soc. Jpn. 82, 102001 (2013).

[5] Y. Ando and L. Fu, Topological Crystalline Insulators and Topological Superconductors: From Concepts to Materials, Annu. Rev. Condens. Matter Phys. 6, 361 (2015).

[6] T. Mizushima, Y. Tsutsumi, T. Kawakami, M. Sato, M. Ichioka, and K. Machida, Symmetry-Protected Topological Superfluids and Superconductors-From the Basics to ${ }^{3} \mathrm{He}$, J. Phys. Soc. Jpn. 85, 022001 (2016).

[7] M. Sato and S. Fujimoto, Majorana Fermions and Topology in Superconductors, J. Phys. Soc. Jpn. 85, 072001 (2016).

[8] M. Sato and Y. Ando, Topological Superconductors: A Review, Rep. Prog. Phys. 80, 076501 (2017).

[9] L. Fu and C. L. Kane, Topological Insulators with Inversion Symmetry, Phys. Rev. B 76, 045302 (2007).

[10] X.-L. Qi, T. L. Hughes, and S.-C. Zhang, Topological Field Theory of Time-Reversal Invariant Insulators, Phys. Rev. B 78, 195424 (2008).

[11] G. E. Volovik, The Universe in a Helium Droplet (Oxford University, New York, 2003).

[12] J. Goryo and K. Ishikawa, Observation of Induced Chern-Simons Term in $P$ - and T-Violating Superconductors, Phys. Lett. A 260, 294 (1999).

[13] N. Read and D. Green, Paired States of Fermions in Two Dimensions with Breaking of Parity and Time-Reversal 
Symmetries and the Fractional Quantum Hall Effect, Phys. Rev. B 61, 10267 (2000).

[14] A. P. Schnyder, S. Ryu, A. Furusaki, and A. W. W. Ludwig, Classification of Topological Insulators and Superconductors in Three Spatial Dimensions, Phys. Rev. B 78, 195125 (2008).

[15] X.-L. Qi, T. L. Hughes, S. Raghu, and S.-C. Zhang, TimeReversal-Invariant Topological Superconductors and Superfluids in Two and Three Dimensions, Phys. Rev. Lett. 102, 187001 (2009).

[16] R. Roy, Topological Superfluids with Time Reversal Symmetry, arXiv:0803.2868.

[17] M. Sato, Topological Properties of Spin-Triplet Superconductors and Fermi Surface Topology in the Normal State, Phys. Rev. B 79, 214526 (2009).

[18] L. Fu and E. Berg, Odd-Parity Topological Superconductors: Theory and Application to $\mathrm{Cu}_{x} \mathrm{Bi}_{2} \mathrm{Se}_{3}$, Phys. Rev. Lett. 105, 097001 (2010).

[19] M. Sato, Topological Odd-Parity Superconductors, Phys. Rev. B 81, 220504 (2010).

[20] S. Sasaki, M. Kriener, K. Segawa, K. Yada, Y. Tanaka, M. Sato, and Y. Ando, Topological Superconductivity in $\mathrm{Cu}_{x} \mathrm{Bi}_{2} \mathrm{Se}_{3}$, Phys. Rev. Lett. 107, 217001 (2011).

[21] L. Fu, Odd-Parity Topological Superconductor with Nematic Order: Application to $\mathrm{Cu}_{x} \mathrm{Bi}_{2} \mathrm{Se}_{3}$, Phys. Rev. B 90, 100509 (2014).

[22] K. Matano, M. Kriener, K. Segawa, Y. Ando, and G.-q. Zheng, Spin-Rotation Symmetry Breaking in the Superconducting State of $\mathrm{Cu}_{x} \mathrm{Bi}_{2} \mathrm{Se}_{3}$, Nat. Phys. 12, 852 (2016).

[23] S. Yonezawa, K. Tajiri, S. Nakata, Y. Nagai, Z. Wang, K. Segawa, Y. Ando, and Y. Maeno, Thermodynamic Evidence for Nematic Superconductivity in $\mathrm{Cu}_{x} \mathrm{Bi}_{2} \mathrm{Se}_{3}$, Nat. Phys. 13, 123 (2017).

[24] Z. Liu, X. Yao, J. Shao, M. Zuo, L. Pi, S. Tan, C. Zhang, and Y. Zhang, Superconductivity with Topological Surface State in $\mathrm{Sr}_{x} \mathrm{Bi}_{2} \mathrm{Se}_{3}$, J. Am. Chem. Soc. 137, 10512 (2015).

[25] Y. Qiu, K. Nocona Sanders, J. Dai, J. E. Medvedeva, W. Wu, P. Ghaemi, T. Vojta, and Y. San Hor, Time Reversal Symmetry Breaking Superconductivity in Topological Materials, arXiv:1512.03519.

[26] Shruti, V. K. Maurya, P. Neha, P. Srivastava, and S. Patnaik, Superconductivity by $\mathrm{Sr}$ Intercalation in the Layered Topological Insulator $\mathrm{Bi}_{2} \mathrm{Se}_{3}$, Phys. Rev. B 92, 020506 (2015).

[27] T. Asaba, B. J. Lawson, C. Tinsman, L. Chen, P. Corbae, G. Li, Y. Qiu, Y. S. Hor, L. Fu, and L. Li, Rotational Symmetry Breaking in a Trigonal Superconductor $\mathrm{Nb}$-doped $\mathrm{Bi}_{2} \mathrm{Se}_{3}$, Phys. Rev. X 7, 011009 (2017).

[28] S. Murakami and N. Nagaosa, Berry Phase in Magnetic Superconductors, Phys. Rev. Lett. 90, 057002 (2003).

[29] G. Y. Cho, J. H. Bardarson, Y.-M. Lu, and J. E. Moore, Superconductivity of Doped Weyl Semimetals: FiniteMomentum Pairing and Electronic Analog of the ${ }^{3} \mathrm{He}-A$ Phase, Phys. Rev. B 86, 214514 (2012).

[30] B. Lu, K. Yada, M. Sato, and Y. Tanaka, Crossed Surface Flat Bands of Weyl Semimetal Superconductors, Phys. Rev. Lett. 114, 096804 (2015).

[31] Y. Li and D. M. Haldane, Topological Nodal Cooper Pairing in Doped Weyl Metals, Phys. Rev. Lett. 120, 067003 (2018).
[32] L. Aggarwal, A. Gaurav, G. S. Thakur, Z. Haque, A. K. Ganguli, and G. Sheet, Mesoscopic Superconductivity and High Spin Polarization Coexisting at Metallic Point Contacts on Weyl Semimetal TaAs, Nat. Mater. 15, 32 (2016).

[33] H. Wang, H. Wang, H. Liu, H. Lu, W. Yang, S. Jia, X.-J. Liu, X. C. Xie, J. Wei, and J. Wang, Observation of Superconductivity Induced by a Point Contact on 3D Dirac Semimetal $\mathrm{Cd}_{3} \mathrm{As}_{2}$ Crystals, Nat. Mater. 15, 38 (2016).

[34] G. Bednik, A. A. Zyuzin, and A. A. Burkov, Superconductivity in Weyl Metals, Phys. Rev. B 92, 035153 (2015).

[35] S. Kobayashi and M. Sato, Topological Superconductivity in Dirac Semimetals, Phys. Rev. Lett. 115, 187001 (2015).

[36] T. Hashimoto, S. Kobayashi, Y. Tanaka, and M. Sato, Superconductivity in Doped Dirac Semimetals, Phys. Rev. B 94, 014510 (2016).

[37] J. Shen, W.-Y. He, N. F. Q. Yuan, Z. Huang, C.-w. Cho, S. H. Lee, Y. S. Hor, K. T. Law, and R. Lortz, Nematic Topological Superconducting Phase in $\mathrm{Nb}$-Doped $\mathrm{Bi}_{2} \mathrm{Se}_{3}$, npj Quantum Mater. 2, 59 (2017).

[38] P. M. R. Brydon, L. Wang, M. Weinert, and D. F. Agterberg, Pairing of $j=3 / 2$ Fermions in Half-Heusler Superconductors, Phys. Rev. Lett. 116, 177001 (2016).

[39] W. Yang, Y. Li, and C. Wu, Topological Septet Pairing with Spin-3/2 Fermions: High-Partial-Wave Channel Counterpart of the ${ }^{3} \mathrm{He}-\mathrm{B}$ Phase, Phys. Rev. Lett. 117, 075301 (2016).

[40] I. Boettcher and I. F. Herbut, Superconducting Quantum Criticality in Three-Dimensional Luttinger Semimetals, Phys. Rev. B 93, 205138 (2016).

[41] L. Savary, J. Ruhman, J. W. F. Venderbos, L. Fu, and P. A. Lee, Superconductivity in Three-Dimensional Spin-Orbit Coupled Semimetals, Phys. Rev. B 96, 214514 (2017).

[42] I. Boettcher and I. F. Herbut, Unconventional Superconductivity in Luttinger Semimetals: Theory of Complex Tensor Order and the Emergence of the Uniaxial Nematic State, Phys. Rev. Lett. 120, 057002 (2018).

[43] B. Roy, S. A. A. Ghorashi, M. S. Foster, and A. H. Nevidomskyy, Topological Superconductivity of Spin-3/2 Carriers in a Three-Dimensional Doped Luttinger Semimetal, arXiv:1708.07825.

[44] C. Timm, A. P. Schnyder, D. F. Agterberg, and P. M. R. Brydon, Inflated Nodes and Surface States in Superconducting Half-Heusler Compounds, Phys. Rev. B 96, 094526 (2017).

[45] Sayed Ali Akbar Ghorashi, S. Davis, and M. S. Foster, Disorder-Enhanced Topological Protection and Universal Quantum Criticality in a Spin- $\frac{3}{2}$ Topological Superconductor, Phys. Rev. B 95, 144503 (2017).

[46] J. W. F. Venderbos, L. Savary, J. Ruhman, P. A. Lee, and L. $\mathrm{Fu}$, Pairing States of Spin-3/2 Fermions: SymmetryEnforced Topological Gap Functions, Phys. Rev. X 8, 011029 (2018).

[47] T.-1. Ho and S. Yip, Pairing of Fermions with Arbitrary Spin, Phys. Rev. Lett. 82, 247 (1999).

[48] I. Kuzmenko, T. Kuzmenko, Y. Avishai, and M. Sato, SpinOrbit Coupling and Topological States in $F=\frac{3}{2}$ Cold Fermi Gas, arXiv:1801.05646 [Phys. Rev. B (to be published)].

[49] J. Yu and C.-X. Liu, Singlet-Quintet Mixing in Spin-Orbit Coupled Superconductors with $j=3 / 2$ Fermions, Phys. Rev. B 98, 104514 (2018). 
[50] T. Kariyado and M. Ogata, Three-Dimensional Dirac Electrons at the Fermi Energy in Cubic Inverse Perovskites: $\mathrm{Ca}_{3} \mathrm{PbO}$ and Its Family, J. Phys. Soc. Jpn. 80, 083704 (2011).

[51] T. Kariyado and M. Ogata, Low-Energy Effective Hamiltonian and the Surface States of $\mathrm{Ca}_{3} \mathrm{PbO}$, J. Phys. Soc. Jpn. 81, 064701 (2012).

[52] T. H. Hsieh, J. Liu, and L. Fu, Topological Crystalline Insulators and Dirac Octets in Antiperovskites, Phys. Rev. B 90, 081112 (2014).

[53] C. Fang and L. Fu, Rotation Anomaly and Topological Crystalline Insulators, arXiv:1709.01929.

[54] M. Oudah, A. Ikeda, J. N. Hausmann, S. Yonezawa, T. Fukumoto, S. Kobayashi, M. Sato, and Y. Maeno, Superconductivity in the Antiperovskite Dirac-Metal Oxide $\mathrm{Sr}_{3-x} \mathrm{SnO}$, Nat. Commun. 7, 13617 (2016).

[55] J. N. Hausmann, M. Oudah, A. Ikeda, S. Yonezawa, and Y. Maeno, Controlled Synthesis of the Antiperovskite Oxide Superconductor $\mathrm{Sr}_{3-x} \mathrm{SnO}$, arXiv:1712.09484.

[56] A. Ikeda, T. Fukumoto, M. Oudah, J. N. Hausmann, S. Yonezawa, S. Kobayashi, M. Sato, C. Tassel, F. Takeiri, H. Takatsu et al., Theoretical Band Structure of the Superconducting Antiperovskite Oxide $\mathrm{Sr}_{3-x} \mathrm{SnO}$, Physica (Amsterdam) 536B, 752 (2018).

[57] M. Oudah, J. N. Hausmann, S. Kitao, A. Ikeda, S. Yonezawa, M. Seto, and Y. Maeno (unpublished).

[58] S. Kitagawa, K. Ishida, M. Oudah, J. N. Hausmann, A. Ikeda, S. Yonezawa, and Y. Maeno, Normal-State Properties of the Antiperovskite Oxide $\mathrm{Sr}_{3-x} \mathrm{SnO}$ Revealed by ${ }^{119}$ Sn-NMR, Phys. Rev. B 98, 100503(R) (2018).

[59] M.S. Foster, H.-Y. Xie, and Y.-Z. Chou, Topological Protection, Disorder, and Interactions: Survival at the Surface of Three-Dimensional Topological Superconductors, Phys. Rev. B 89, 155140 (2014).

[60] W. J. Elder, R. M. Ward, and J. Zhang, Double-Group Formulation of $\mathbf{k} \cdot \mathbf{p}$ Theory for Cubic Crystals, Phys. Rev. B 83, 165210 (2011).

[61] In the strong pairing case, the large gap function may mix the contributions from each band, but their summation is conserved.

[62] X.-L. Qi, T. L. Hughes, and S.-C. Zhang, Topological Invariants for the Fermi Surface of a Time-ReversalInvariant Superconductor, Phys. Rev. B 81, 134508 (2010).

[63] We observe that $\left|w_{\text {out }}\right|$ changes only by 2 at $v_{2} / v_{1}=1 / 2$, although there appear eight point nodes on the outer Fermi surface, which is because the inner and outer Fermi surfaces contact each other at $v_{2} / v_{1}=1 / 2$, and, hence, they exchange their winding numbers.
[64] C.-K. Chiu, H. Yao, and S. Ryu, Classification of Topological Insulators and Superconductors in the Presence of Reflection Symmetry, Phys. Rev. B 88, 075142 (2013).

[65] T. Morimoto and A. Furusaki, Topological Classification with Additional Symmetries from Clifford Algebras, Phys. Rev. B 88, 125129 (2013).

[66] K. Shiozaki and M. Sato, Topology of Crystalline Insulators and Superconductors, Phys. Rev. B 90, 165114 (2014).

[67] K. Shiozaki, M. Sato, and K. Gomi, Topology of Nonsymmorphic Crystalline Insulators and Superconductors, Phys. Rev. B 93, 195413 (2016).

[68] K. Shiozaki, M. Sato, and K. Gomi, Topological Crystalline Materials: General Formulation, Module Structure, and Wallpaper Groups, Phys. Rev. B 95, 235425 (2017).

[69] Y. Ueno, A. Yamakage, Y. Tanaka, and M. Sato, SymmetryProtected Majorana Fermions in Topological Crystalline Superconductors: Theory and Application to $\mathrm{Sr}_{2} \mathrm{RuO}_{4}$, Phys. Rev. Lett. 111, 087002 (2013).

[70] T. Mizushima and K. Machida, Splitting and Oscillation of Majorana Zero Modes in the p-Wave BCS-BEC Evolution with Plural Vortices, Phys. Rev. A 82, 023624 (2010).

[71] M. Sigrist and K. Ueda, Phenomenological Theory of Unconventional Superconductivity, Rev. Mod. Phys. 63, 239 (1991).

[72] A. Yamakage, K. Yada, M. Sato, and Y. Tanaka, Theory of Tunneling Conductance and Surface-State Transition in Superconducting Topological Insulators, Phys. Rev. B 85, 180509 (2012).

[73] In antiperovskites, the opposite parities of two $J=3 / 2$ states originate from those of the atomic orbitals of $A$ and $B$. However, in general, not only the atomic orbital d.o.f. but also sublattice ones can form the two states with different parities discussed here [74]. In fact, in the spin-1/2 TIs of the $\mathrm{Bi}_{2} \mathrm{Se}_{3}$ family, orbital states with odd and even parity both originate from $p$ orbitals forming bonding and antibonding states between different sublattices. The two $J=3 / 2$ states with opposite parity can also be obtained through this mechanism, in general.

[74] J. Cano, B. Bradlyn, Z. Wang, L. Elcoro, M. G. Vergniory, C. Felser, M. I. Aroyo, and B. A. Bernevig, Topology of Disconnected Elementary Band Representations, Phys. Rev. Lett. 120, 266401 (2018).

[75] C. Fang, M. J. Gilbert, and B. A. Bernevig, New Class of Topological Superconductors Protected by Magnetic Group Symmetries, Phys. Rev. Lett. 112, 106401 (2014).

[76] A. L. Fetter and J. D. Walecka, Quantum Theory of ManyParticle Systems (Dover, New York, 1971). 